INRA Prod. Anim., 2006, 19 (1), 15-28

\title{
Relations entre les conditions de production du lait et les teneurs en composés d'intérêt nutritionnel dans le fromage : étude en conditions réelles de production
}

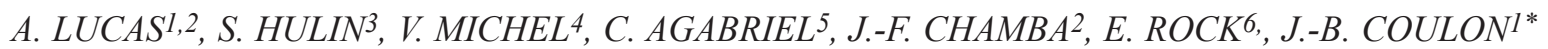 \\ ${ }^{1}$ INRA, Unité de Recherches sur les Herbivores, F-63122 Saint-Genès Champanelle \\ 2 ITFF, 419 route des Champs Laitiers, BP 30, F-74801 La Roche sur Foron \\ 3 Pôle Fromager AOC Massif Central, 21 rue du Château Saint-Etienne, F-15000 Aurillac \\ ${ }^{4}$ GIS Alpes du Nord, 11 rue Métropole, F-73000 Chambéry \\ ${ }^{5}$ ENITAC, Marmilhat, F-63370 Lempdes \\ ${ }^{6}$ INRA, Unité Maladies Métaboliques et Micronutriments, F-63122 Saint-Genès Champanelle \\ *Courriel: jbc@clermont.inra.fr
}

Les caractéristiques nutritionnelles du fromage font l'objet de nombreuses interrogations de la part des filières fromagères notamment quant à leur lien avec les pratiques d'élevage. Certains travaux suggèrent que ces caractéristiques dépendent de facteurs de production liés notamment à l'alimentation des animaux. Les conditions de la transformation fromagère semblent également pouvoir modifier ces caractéristiques. Mais quelle est l'importance relative des pratiques d'élevage et de transformation fromagère, et quel est le rôle des différents facteurs de production du lait sur les caractéristiques nutritionnelles du fromage en conditions réelles de production?

\begin{abstract}
Il est désormais admis que l'alimentation joue un rôle sur la santé humaine et notamment sur le développement des maladies chroniques (Darnton-Hill et al 2004). Une meilleure compréhension de cette relation implique de disposer de solides connaissances de la qualité nutritionnelle des aliments. Celle des produits laitiers dépend à la fois de leurs caractéristiques biochimiques (teneurs en macro et micronutriments, voire en divers contaminants) et microbiologiques (présence éventuelle de microorganismes probiotiques). La composition du fromage en ces éléments ainsi que ses facteurs de variation souffrent actuellement d'un manque d'information. C'est notamment le cas des teneurs du fromage en acides gras $(\mathrm{AG})$, vitamines, minéraux et antioxydants, qui fait l'objet de ce travail. Combler ce manque d'information s'avère cependant complexe en raison du caractère multifactoriel de l'élaboration des caractéristiques nutritionnelles du fromage. Ainsi, la compo-
\end{abstract}

sition du lait à partir duquel le fromage est fabriqué varie selon ses conditions de production et plus précisément selon la nature de l'alimentation et les caractéristiques des animaux. La nature de la ration de base des animaux ainsi que le niveau et la nature des concentrés semblent être des facteurs de variation important de la composition du lait en AG, vitamines et caroténoïdes (Agabriel et al 2004, Martin et al 2002, Chilliard et al 2000, Sharma et al 1983, Stockdale et al 2003). Les caractéristiques des animaux (l'espèce, la race et le stade de lactation) sont également des facteurs de variation de ces teneurs en composés d'intérêt nutritionnel (Chilliard et al 2000, Guéguen 1971, Jensen et al 1999, Krukovsky et al 1950, Lawless et al 1999, Sollberger et al 2004). D'autre part, au cours de la transformation fromagère, certains paramètres technologiques sont susceptibles de modifier de façon plus ou moins importante les caractéristiques nutritionnelles du lait et donc d'influen- cer celles du fromage. C'est par exemple le cas du niveau d'acidification du lait avant l'étape d'égouttage pour les minéraux (Lucey et Fox 1993) ou de la nature des microorganismes pour les vitamines B (Shahani et al 1962).

Bien que les principaux facteurs de production susceptibles d'influencer les caractéristiques nutritionnelles du fromage semblent d'une manière générale bien identifiés, les données disponibles dans la littérature présentent plusieurs limites. Tout d'abord, l'influence des conditions de production du lait a principalement été étudiée sur les caractéristiques du lait et rarement directement sur celles du fromage. Or, en raison d'un manque de données concernant les effets de la transformation fromagère sur la composition du fromage, il est difficile de savoir dans quelle mesure les informations relatives au lait sont extrapolables au fromage. D'autre part, la plupart des études réalisées jusqu'à présent, généralement en conditions 
expérimentales, ne se sont intéressées qu'aux effets d'un nombre restreint de facteurs de production sur la composition du lait en quelques micronutriments. Elles ne permettent donc pas de disposer d'une vision globale de l'importance relative des différents facteurs de production sur les teneurs du fromage en une large gamme de composés d'intérêt nutritionnel.

Dans ce contexte, les objectifs de cette étude ont été de : 1/ caractériser la composition en composés d'intérêt nutritionnel de plusieurs variétés de fromages fermiers représentant différentes technologies fromagères ; 2/ quantifier les effets respectifs des conditions de production du lait et de la transformation fromagère sur la composition du fromage de façon à identifier les composés dont les variations dans le fromage sont significativement attribuables aux conditions de production du lait; et $3 /$ relier les variations des teneurs en composés d'intérêt nutritionnel du fromage à ces conditions de production du lait. $\mathrm{Ce}$ travail, réalisé dans le cadre des programmes de recherche et développement du Groupement d'Intérêt Scientifique (GIS) Alpes du Nord et du Pôle Fromager AOC Massif Central, a fait l'objet de plusieurs publications (Lucas 2005, Lucas et al 2006a, 2006b) dont ce texte fait la synthèse.

\section{1 / Description de l'étude}

Cette étude a été conduite entre août 2002 et décembre 2004 au sein de 4 filières de fromages au lait cru : Abondance (pâte pressée mi-cuite, lait de vache) et Tomme de Savoie (pâte pressée non cuite, lait de vache) dans les Alpes du Nord, Salers/Cantal (pâte pressée non cuite, lait de vache) et Rocamadour (coagulation lactique, lait de chèvre) dans le Massif Central. Au total, 74 producteurs fermiers ont participé à ce travail (16 à 20 par variété fromagère).

Dans un premier temps, une préenquête (ENITA, 2002) a été réalisée auprès de chaque producteur, de façon à valider l'existence d'une diversité de conditions de production du lait satisfaisante au sein de l'échantillon de producteurs préalablement sélectionné par les filières fromagères. Les informations recueillies concernaient notamment la nature de l'alimentation (fourrages, concentrés, compléments) et les caractéristiques (race, niveau de production, périodes de mise bas, taux de renouvellement) des animaux. Dans un second temps, 6 à 7 fromages produits tout au long de l'année ont été prélevés chez chacun de ces producteurs. Au total, 432 fromages, dont 306 au lait de vache et 126 au lait de chèvre, ont été collectés. En amont de ces prélèvements, une enquête a été réalisée le jour de la fabrication des fromages de façon à identifier les conditions de production spécifiques à chaque fromage prélevé. Cette enquête s'est intéressée tout particulièrement aux facteurs de production susceptibles de varier de façon saisonnière (alimentation, stade de lactation, niveau de production...). D'autre part, de façon à quantifier l'influence de la transformation fromagère sur la composition du fromage, 53 échantillons de lait (10 à 18 par variété fromagère) correspondant à 53 des fromages prélevés ont également été collectés.

Pour chaque échantillon de lait et de fromage, nous avons analysé, en plus des taux de matières sèches, de matières grasses et de protéines, le profil en $A G$, les teneurs en vitamines $\mathrm{A}$, $\mathrm{E}$ et $\mathrm{B} 9$, en $\beta$-carotène, xanthophylles, calcium, phosphore, magnésium, potassium, zinc et le potentiel antioxydant total. La teneur en chlorure de sodium a également été mesurée mais seulement dans le fromage. Le traitement statistique des données a consisté tout d'abord à quantifier l'influence relative de la transformation fromagère et de la composition $\mathrm{du}$ lait sur la composition du fromage par analyse de variance-covariance. Puis, les relations entre les conditions de production du lait et la composition du fromage ont été analysées en ne considérant que les composés dont les teneurs dans le fromage dépendent significativement de celles du lait d'origine et en étudiant séparément les fromages au lait de vache et ceux au lait de chèvre. Pour cela, une analyse factorielle des correspondances multiples a été réalisée dans un premier temps de façon à disposer d'une vision globale des associations existant entre les facteurs de production et les caractéristiques du fromage. Dans un second temps, les effets respectifs des différents facteurs de production ont été quantifiés par analyse de variance. Enfin, une typologie des fromages a été réalisée par classification ascendante hiérarchique sur la base de leurs teneurs en composés d'intérêt nutritionnel.

\section{2 / Variabilité des teneurs en composés d'intérêt nu- tritionnel dans le fromage (Lucas 2005, Lucas et al 2006a)}

Cette étude a permis de caractériser les teneurs en composés d'intérêt nutritionnel des 4 variétés fromagères étudiées en considérant un nombre suffisamment important d'échantillons pour disposer d'une représentativité satisfaisante. Elle a mis en évidence l'existence d'une forte variabilité de composition d'une variété de fromage à l'autre, qui ne dépend pas uniquement des différences de teneur en matière sèche (tableau 1). Par exemple, le Rocamadour est de 4 à 6 fois plus riche en vitamine B9, mais de 6 à 8 fois moins riche en calcium et en zinc que l'Abondance, la Tomme de Savoie et les fromages de type Cantal. De même, il est dépourvu de $\beta$-carotène contrairement aux autres variétés fromagères.

Il existe également une forte variabilité intra variété fromagère (tableau 1). Cette variabilité dépend de la nature des composés. Ainsi, les vitamines, les caroténoïdes et certains AG mineurs tels que l'acide ruménique (C18:2 cis9 trans-11), l'isomère majoritaire des acides linoléiques conjugués (CLA) dans le fromage, varient fortement dans les 4 variétés fromagères étudiées. Par exemple, entre le $5^{\mathrm{e}}$ et le $95^{\mathrm{e}}$ centiles, la teneur en vitamine $\mathrm{E}$ varie en moyenne d'un facteur 1 à 4,5 et celle en CLA d'un facteur 1 à 3,7 intra variété fromagère. En revanche, les minéraux et les acides gras majeurs varient relativement peu. Par exemple, la teneur en calcium ne varie en moyenne que d'un facteur 1 à 1,5 et celle en acide oléique (C18:1 cis-9) que d'un facteur 1 à 1,7 entre le $5^{\mathrm{e}}$ et le $95^{\mathrm{e}}$ centiles intra variété fromagère. D'autre part, le niveau de variation de certains composés dépend du type de fromage. Par exemple, l'acide linolénique (C18:3 n-3) varie davantage dans le Rocamadour que dans les 3 autres variétés fromagères, tandis que le contraire a été observé pour la teneur en vitamine B9.

Les variations de ces teneurs en composés d'intérêt nutritionnel ont des conséquences importantes sur la contribution de la consommation de fromages à la couverture des apports nutritionnels conseillés (tableau 1). Ainsi, 
Tableau 1. Teneurs en composés d'intérêt nutritionnel dans le fromage (Lucas 2005, Lucas et al 2006a).

\begin{tabular}{|c|c|c|c|c|c|c|c|c|c|}
\hline & \multicolumn{4}{|c|}{ Teneurs moyennes $^{1,2}$} & \multicolumn{4}{|c|}{$95^{\mathrm{e}} / 5^{\mathrm{e}}$ centile $^{2,3}$} & \multirow{2}{*}{$\begin{array}{c}\% \\
\text { ANC } \\
(40 \mathrm{~g})^{4}\end{array}$} \\
\hline & A & $\mathrm{T}$ & $\mathrm{S} / \mathrm{C}$ & $\mathrm{R}$ & A & $\mathrm{T}$ & $\mathrm{S} / \mathrm{C}$ & $\mathrm{R}$ & \\
\hline \multicolumn{10}{|l|}{ Composition brute } \\
\hline Matières sèches $\left(\mathrm{g} \cdot \mathrm{kg}^{-1}\right)$ & 638 & 585 & 609 & 434 & 1,1 & 1,2 & 1,1 & 1,3 & - \\
\hline Matières grasses $\left(\mathrm{g} \cdot \mathrm{kg}^{-1}\right)$ & 331 & 302 & 306 & 232 & 1,2 & 1,2 & 1,2 & 1,4 & - \\
\hline Gras / Sec (\%) & 52 & 52 & 50 & 53 & 1,1 & 1,1 & 1,2 & 1,2 & - \\
\hline \multicolumn{10}{|l|}{ Acides gras $\left(\mathrm{g} \cdot \mathrm{kg}^{-1}\right)$} \\
\hline Saturés & 202 & 183 & 187 & 149 & 1,3 & 1,3 & 1,3 & 1,4 & $22-49$ \\
\hline $\mathrm{C} 4: 0$ & 12 & 11 & 12 & 5,7 & 1,5 & 1,5 & 1,5 & 1,8 & - \\
\hline C6:0-C10:0 & 22 & 21 & 22 & 34 & 1,7 & 1,5 & 1,5 & 1,8 & - \\
\hline C12:0-C16:0 & 131 & 118 & 118 & 80 & 1,4 & 1,5 & 1,4 & 1,5 & - \\
\hline C18:0 & 24 & 22 & 24 & 21 & 1,6 & 1,8 & 2,0 & 2,7 & - \\
\hline Monoinsaturés & 74 & 68 & 69 & 45 & 1,6 & 1,6 & 1,5 & 1,8 & - \\
\hline C18:1 c9 (n-9) & 51 & 47 & 48 & 35 & 1,6 & 1,7 & 1,7 & 1,8 & $1,5-7,2$ \\
\hline $\mathrm{C} 18: 1 \mathrm{t11}(+t 10)$ & 6,6 & 6,5 & 6,0 & 2,2 & 3,4 & 3,7 & 4,2 & 3,4 & - \\
\hline Polyinsaturés & 13 & 12 & 9,8 & 8,4 & 1,8 & 2,2 & 1,8 & 2,2 & - \\
\hline C18:2 c9c12 (n-6) & 4,9 & 4,1 & 3,3 & 4,6 & 1,6 & 1,7 & 2,0 & 2,0 & $0,8-3,3$ \\
\hline C18:2 c9t11 (CLA) $)^{5}$ & 2,8 & 2,8 & 2,6 & 0,84 & 3,5 & 4,4 & 4,0 & 3,0 & - \\
\hline C18:3 c9c12c15 (n-3) & 2,4 & 2,2 & 1,9 & 1,5 & 2,0 & 2,8 & 2,7 & 3,7 & $0,9-9,4$ \\
\hline Trans (CLA non inclus) & 12 & 11 & 13 & 5,1 & 2,8 & 3,2 & 2,5 & 2,7 & $2,4-19$ \\
\hline \multicolumn{10}{|l|}{ Vitamines et caroténoïdes } \\
\hline$\beta$-Carotène $\left(\mathrm{mg} \cdot \mathrm{kg}^{-1}\right)$ & 1,0 & 1,1 & 1,4 & 0,0 & 5,4 & 6,7 & 4,8 & - & - \\
\hline Xanthophylles $\left(\mu \mathrm{g} \cdot \mathrm{kg}^{-1}\right)$ & 72 & 92 & 93 & 36 & 4,2 & 4,2 & 3,2 & 5,5 & - \\
\hline Rétinol (mg.kg $\left.{ }^{-1}\right)$ & 1,7 & 1,6 & 1,6 & 1,5 & 2,7 & 2,7 & 1,8 & 3,2 & - \\
\hline Vitamine $\mathrm{A}$ total $\left(\times 10^{3} \mathrm{ER} \cdot \mathrm{kg}^{-1}\right)$ & 1,9 & 1,8 & 1,9 & 1,5 & 2,6 & 2,6 & 1,8 & 3,2 & $3,6-25$ \\
\hline Vitamine $\mathrm{E}\left(\mathrm{mg} \cdot \mathrm{kg}^{-1}\right)$ & 1,7 & 1,9 & 2,1 & 1,6 & 4,6 & 5,5 & 3,7 & 4,3 & $0,1-1,6$ \\
\hline Vitamine B9 $\left(\mu \mathrm{g} . \mathrm{kg}^{-1}\right)$ & 176 & 269 & 178 & 1010 & 4,6 & 2,7 & 3,6 & 1,7 & $0,8-18$ \\
\hline \multicolumn{10}{|l|}{ Minéraux } \\
\hline Chlorure de sodium $\left(\mathrm{g} \cdot \mathrm{kg}^{-1}\right)$ & 16 & 15 & 24 & 11 & 2,3 & 2,3 & 1,8 & 1,6 & $3,5-22$ \\
\hline Calcium $\left(\mathrm{g} \cdot \mathrm{kg}^{-1}\right)$ & 8,8 & 6,6 & 8,1 & 1,1 & 1,5 & 1,6 & 1,3 & 1,8 & $3,2-53$ \\
\hline Phosphore (g.kg-1) & 5,5 & 4,6 & 5,0 & 2,2 & 1,2 & 1,2 & 1,1 & 1,3 & $9,7-32$ \\
\hline Potassium $\left(\mathrm{g} \cdot \mathrm{kg}^{-1}\right)$ & 1,0 & 0,9 & 1,0 & 1,6 & 1,4 & 1,7 & 1,5 & 1,4 & $4,0-17$ \\
\hline Magnésium (mg.kg $\left.{ }^{-1}\right)$ & 322 & 249 & 289 & 160 & 1,5 & 1,6 & 1,6 & 1,7 & $1,0-4,4$ \\
\hline Zinc (mg. $\left.\mathrm{kg}^{-1}\right)$ & 46 & 33 & 36 & 5,7 & 1,5 & 1,7 & 1,6 & 1,6 & $1,3-28$ \\
\hline Pot. antiox. ${ }^{6}\left(\mathrm{~mol} \mathrm{Fe}^{2+} \cdot \mathrm{kg}^{-1}\right)$ & 8,1 & 7,5 & 9,1 & 5,1 & 2,0 & 2,7 & 2,2 & 4,1 & - \\
\hline
\end{tabular}

1 Exprimées par kg de produit frais. ${ }^{2}$ A: Abondance $(n=92)$; T: Tomme de Savoie $(n=105)$; S/C: Salers/Cantal $(n=109)$; R: Rocamadour $(n=126) .{ }^{3}$ Rapport du $95^{e}$ centile sur le $5^{\text {e }}$ centile. 4 Plage de variation mesurée au sein des 432 fromages analysés de la contribution (\%) aux recommandations nutritionnelles minimales pour le potassium et maximales pour les acides gras trans et le chlorure de sodium et aux apports nutritionnels conseillés d'un homme adulte pour les autres nutriments (Martin 2001, AFSSA 2002, AFSSA 2005) de la consommation quotidienne de $40 \mathrm{~g}$ de fromage, ce qui correspond à la quantité moyenne de fromage affiné consommée par habitant et par jour en France (CNIEL 2005). Pour les acides gras, une consommation quotidienne d'énergie de 9,2 MJ a été considérée. ${ }^{5}$ CLA : Acide linoléique conjugué. ${ }^{6}$ Pot. antiox. : potentiel antioxydant.

au sein des 432 fromages analysés, la contribution de la consommation de $40 \mathrm{~g}$ de fromage à la couverture des apports nutritionnels conseillés d'un

\section{3 / Effets respectifs de la transformation fromagère et des conditions de produc- tion du lait sur les caracté- ristiques nutritionnelles du fromage (Lucas et al 2006b)}

La comparaison de la composition des échantillons de fromage avec celle des échantillons de lait correspondants nous a permis de quantifier les effets respectifs de la transformation fromagère et des conditions de production du lait sur la composition du fromage. $\mathrm{Ce}$ travail a montré que, selon la nature des composés, la composition du fromage dépend soit majoritairement de la composition initiale du lait et donc des conditions de production de celui-ci, soit exclusivement de la transformation fromagère. Ainsi, les teneurs en composés liposolubles ( $\mathrm{AG}$, vitamines A et $\mathrm{E}$, caroténoïdes) dépendent directement et principalement de celles initialement présentes dans le lait et donc des conditions de production de celui-ci comme cela est illustré pour les acides palmitique et ruménique, le $\beta$-carotène et la vitamine $\mathrm{E}$ dans la figure 1. L'influence de la transformation fromagère sur la composition du fromage en ces composés est par conséquent relativement faible.

En revanche, les teneurs en composés hydrosolubles (minéraux, vitamine B9) dépendent fortement de la transformation fromagère (figure 1). En effet, nous n'avons observé aucune corrélation pour ces composés entre la composition du fromage et celle du lait. Ces résultats confirment ceux de la littérature (Moreno-Rojas et al 1995, Shahani et al 1962, Wong et al 1976). Ainsi, pour un lait de composition initiale identique, le fromage à coagulation lactique (Rocamadour) présente des teneurs plus faibles en calcium, phosphore, magnésium et zinc et une teneur en vitamine B9 plus forte que les fromages à pâte pressée mi-cuite ou non cuite (figure 1). Le type de coagulation du lait (acide pour le Rocamadour, enzymatique pour les autres fromages) explique ces différences. Une coagulation acide conduit en effet à une solubilisation et donc à une perte importante de minéraux dans le lactosérum (Lucey et Fox 1993). D'autre part, la plus forte teneur en vitamine B9 du Rocamadour suggère un niveau de synthèse microbienne pour cette vitamine plus important au cours de la fabrication 
Figure 1. Relations entre la composition du fromage et celle du lait en acide palmitique (C16:0), acide ruménique (CLA), $\beta$-carotène, vitamine $E$, vitamine B9 et calcium. Les résultats sont exprimés pour le C16:0 et le CLA en \% des acides gras totaux, pour le $\beta$-carotène et la vitamine $E$ en $\mathrm{mg}^{\mathrm{kg}} \mathrm{kg}^{-1}$ de matières grasses, pour la vitamine B9 en mg. $\mathrm{kg}^{-1}$ d'eau et pour le calcium en $\mathrm{g} \mathrm{kg}^{-1}$ de protéines (Lucas et al 2006b).
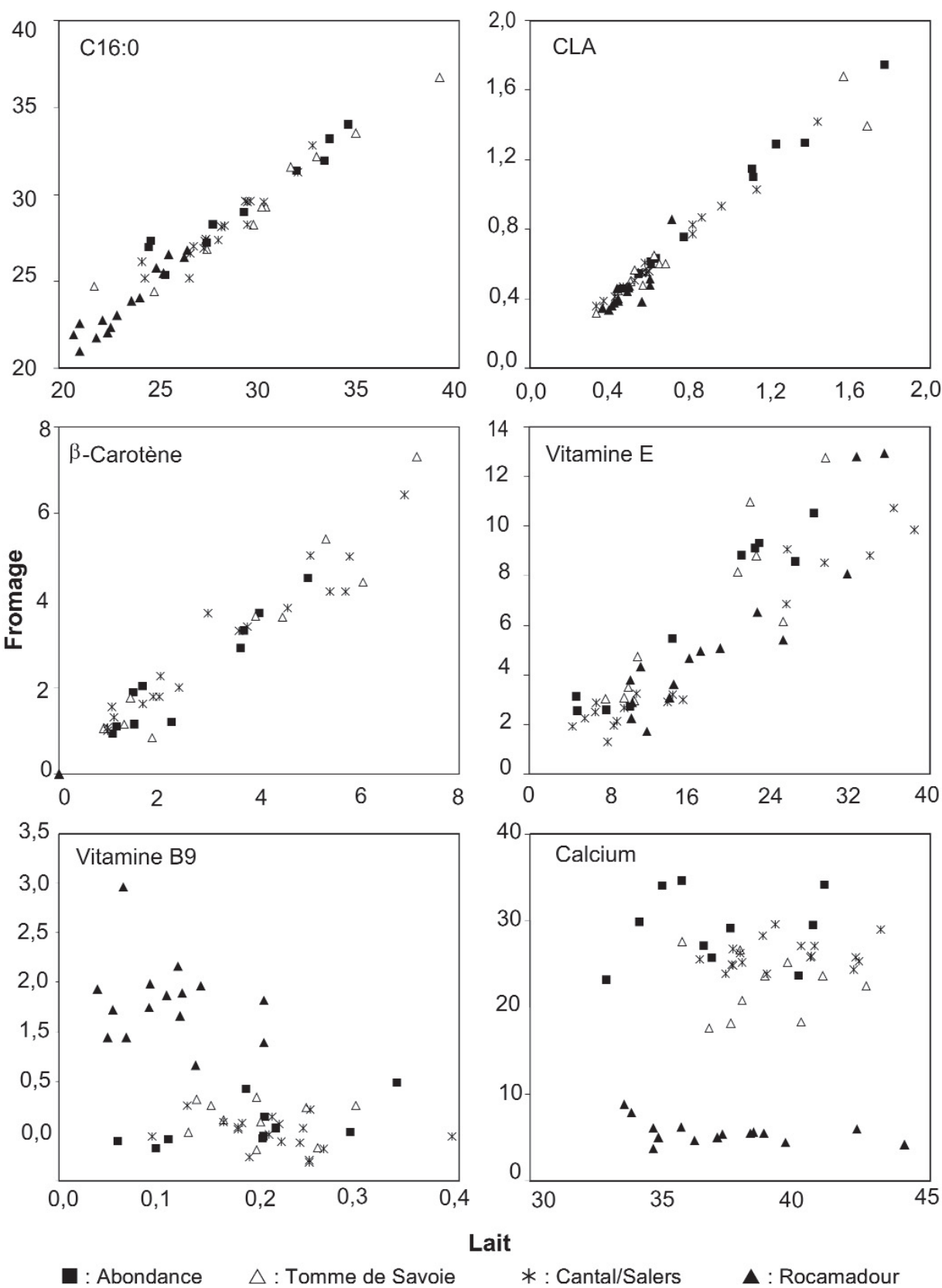

de ce fromage en raison de la nature spécifique de sa microflore (Shahani et al 1962).

Dans la suite de cette étude, les relations entre les conditions de production du lait et les teneurs en composés d'intérêt nutritionnel dans le fromage ont donc été étudiées en considérant uniquement les composés liposolubles dont les teneurs dans le fromage dépendent significativement des conditions de production du lait. en $\beta$-carotène (tableau 2). L'absence totale de $\beta$-carotène dans le fromage au lait de chèvre, à l'inverse de ceux au lait de vache, s'explique en grande partie par une plus forte activité de conversion du $\beta$-carotène en rétinal dans l'intestin grêle des caprins par rapport à celui des bovins (Mora et al 2000, Yang et Tume 1993). Des teneurs plus importantes en vitamines $A$ et $E$ ont en revanche été mesurées dans la matière grasse (MG) des fromages au lait de chèvre (tableau 2). Il est cependant important de souligner que la variabilité intraespèce est plus importante que la variabilité inter-espèces pour ces deux vitamines.

Chez les deux espèces considérées, le mode de conservation des fourrages (pâturage $v s$ fourrages conservés) est apparu comme le principal facteur de variation des teneurs en micronutriments liposolubles ainsi que du potentiel antioxydant du fromage. D'une manière générale, le fromage est d'autant plus riche en vitamines $\mathrm{A}$ et $\mathrm{E}$ et en caroténoïdes que la proportion d'herbe pâturée dans la ration est importante (tableaux 3 et 4), ce qui confirme les résultats expérimentaux obtenus sur le lait de vache (Martin et al 2002) ou de chèvre (Fedele et al 2004). Ces résultats sont dus à la plus grande richesse en vitamine $\mathrm{E}$ et en caroténoïdes de l'herbe verte comparee aux fourrages conservés (Iwanska et al 1997, Robowsky et Knabe 1972).

Les effets d'autres caractéristiques de la ration fourragère ont également été mis en évidence, mais uniquement chez les vaches. Ainsi, lorsque les vaches sont au pâturage, les teneurs en caroténoïdes du fromage semblent également dépendre dans une moindre mesure du stade de développement et de la composition botanique de l'herbe. Les fromages ont en effet présenté des teneurs en caroténoïdes plus importantes lorsque les animaux pâturaient une prairie permanente en première utilisation comparée à des repousses (tableau 3). La plus grande richesse en caroténoïdes de l'herbe à un stade précoce de développement (Park et al 1983) peut en partie expliquer ce résultat. Par ailleurs, les plus faibles teneurs en caroténoïdes des fromages associés au pâturage en alpage comparés à ceux fabriqués à partir du lait d'animaux pâturant en moyenne montagne (tableau 3) pourraient résulter des différences de composition botanique des prairies selon leur altitude (Bugaud et al 2000) et des variations des teneurs en caroté- 
Tableau 2. Caractéristiques nutritionnelles du fromage en fonction de l'espèce anima$\mathrm{le}^{1}$ et en considérant une gamme de conditions de production du lait similaire ${ }^{2}$ entre les deux espèces (Lucas 2005).

\begin{tabular}{|c|c|c|}
\hline Espèce animale & Vache & Chèvre \\
\hline Nombre de fromages & 148 & 55 \\
\hline \multicolumn{3}{|l|}{$\begin{array}{l}\text { Nature de la ration fourragère } \\
\text { (\% des fromages) }\end{array}$} \\
\hline Pâturage & 62 & 27 \\
\hline Fourrages conservés & 38 & 73 \\
\hline \multicolumn{3}{|l|}{ Complémentation en concentrés } \\
\hline $\begin{array}{l}\text { Niveau de complémentation ( } \% \text { de la } \\
\text { ration) }\end{array}$ & $31(20-49)$ & $34(21-50)$ \\
\hline $\begin{array}{l}\text { Matières grasses apportées (\% de la } \\
\text { ration) }\end{array}$ & $0,8(0,5-1,4)$ & $1,0(0,5-1,4)$ \\
\hline Présence de lin (\% des fromages) & 0 & 0 \\
\hline $\begin{array}{l}\text { Complémentation en vitamines } \\
\text { (\% des fromages) }\end{array}$ & 66 & 65 \\
\hline Stade de lactation (mois) ${ }^{3}$ & $5,5(2,9-7,7)$ & $5,4(2,5-7,8)$ \\
\hline \multicolumn{3}{|l|}{ Acides gras (\% des AG totaux) ${ }^{4}$} \\
\hline Saturés & $71^{\mathrm{a}}$ & $73^{b}$ \\
\hline $\mathrm{C} 4: 0$ & $4,2^{b}$ & $2,8^{a}$ \\
\hline C6:0-C10:0 & $8,1^{a}$ & $17^{\mathrm{b}}$ \\
\hline C12:0-C16:0 & $46^{b}$ & $39^{a}$ \\
\hline C18:0 & $7,9^{a}$ & $9,9^{b}$ \\
\hline Monoinsaturés & $24^{b}$ & $22^{\mathrm{a}}$ \\
\hline C18:1 c9 (n-9) & 17 & 18 \\
\hline $\mathrm{C} 18: 1 t 11(+t 10)$ & $1,9^{b}$ & $1,2^{\mathrm{a}}$ \\
\hline Polyinsaturés & 3,9 & 3,9 \\
\hline C18:2 c9c12 (n-6) & $1,5^{\mathrm{a}}$ & $2,2^{b}$ \\
\hline C18:2 c9t11 (CLA) $)^{5}$ & $0,82^{b}$ & $0,46^{a}$ \\
\hline $\mathrm{C} 18: 3 \mathrm{c} 9 \mathrm{c} 12 \mathrm{c} 15(\mathrm{n}-3)$ & $0,75^{\mathrm{b}}$ & $0,65^{a}$ \\
\hline Trans (CLA non inclus) & $3,7^{b}$ & $2,5^{\mathrm{a}}$ \\
\hline \multicolumn{3}{|l|}{ Vitamines et caroténoïdes $^{6}$} \\
\hline$\beta$-Carotène (mg.kg $\left.{ }^{-1} \mathrm{MG}\right)$ & $3,3^{b}$ & $0,0^{a}$ \\
\hline Xanthophylles (mg. $\mathrm{kg}^{-1} \mathrm{MG}$ ) & $0,25^{\mathrm{b}}$ & $0,18^{a}$ \\
\hline Vitamine A (mg.kg $\left.{ }^{-1} \mathrm{MG}\right)$ & $5,2^{\mathrm{a}}$ & $6,8^{b}$ \\
\hline Vitamine $\mathrm{E}$ (mg.kg $\left.{ }^{-1} \mathrm{MG}\right)$ & $5,3^{a}$ & $7,4^{b}$ \\
\hline
\end{tabular}

${ }^{1}$ Moyennes ajustées pour les autres facteurs de production. 2 Une sélection des fromages a été réalisée de façon à comparer des fromages associés à une gamme de conditions de production similaire chez les 2 espèces en termes de nature de la ration fourragère, de niveau de complémentation en concentrés, de proportion de matières grasses apportées par les concentrés et de stade de lactation des animaux. ${ }^{3}$ Moyenne (minimum-maximum). ${ }^{4}$ AG : acides gras. ${ }^{5}$ CLA : acide linoléique conjugué. ${ }^{6} \mathrm{MG}$ : matières grasses. $\mathrm{a}, \mathrm{b}$ : Les valeurs ne présentant aucune lettre en commun diffèrent significativement $(P<0,05)$

noïdes entre espèces végétales (Park et al 1983).

La variabilité des teneurs en micronutriments liposolubles du fromage est moins importante lorsque l'alimentation des vaches est à base de fourrages conservés (tableau 3 ). Les rations à base d'herbe fermentée ont cepen- ainsi qu'à la pauvreté en $\beta$-carotène de l'ensilage de maïs.

La composition en micronutriments liposolubles $\mathrm{du}$ fromage au lait de vache dépend également dans une certaine mesure du niveau de complémentation en concentrés, mais uniquement lorsque la ration des animaux est à base de pâturage. Dans ces conditions, les teneurs en vitamine A et en xanthophylles du fromage sont d'autant plus faibles que le niveau de complémentation en concentrés est élevé. Au cours de cette étude, elles étaient ainsi égales à 0,35 et 0,27 mg. $\mathrm{kg}^{-1} \mathrm{MG}(P<0,001)$ pour les xanthophylles et à 5,76 et $5,26 \mathrm{mg} \cdot \mathrm{kg}^{-1} \mathrm{MG}(P<0,05)$ pour la vitamine A, lorsque la proportion de concentrés dans la ration était respectivement de l'ordre de $15 \%$ et de $41 \%$. Ces résultats reflètent une plus faible consommation d'herbe verte lorsque la quantité de concentrés consommés augmente ainsi qu'une plus grande richesse en caroténoïdes de l'herbe verte comparée aux concentrés. En revanche, probablement en raison de la pauvreté en caroténoïdes des fourrages conservés, il semble que le niveau de complémentation en concentrés n'influe pas sur la quantité de ces composés consommée par les animaux et donc sur leur niveau dans le fromage lorsque des rations à base de fourrages conservés sont distribuées aux animaux.

D'autre part, la teneur en vitamine A et dans une moindre mesure celle en vitamine $\mathrm{E}$ des fromages au lait de vache fabriqués en période hivernale varient directement avec le niveau de supplémentation vitaminique de l'alimentation des animaux, ce qui n'est pas le cas en période de pâturage (figure 2). Des résultats similaires ont été obtenus chez la chèvre. Ces résultats suggèrent donc que la quantité de provitamine $\mathrm{A}$ et de vitamine $\mathrm{E}$ apportée aux animaux par l'herbe verte est suffisante pour permettre une sécrétion maximale de vitamines A et $\mathrm{E}$ dans le lait.

Enfin, parmi les caractéristiques des animaux (race, stade de lactation et parité), dans notre étude, seule la race constitue un facteur de variation important de la composition du fromage en micronutriments liposolubles. Elle a ainsi été associée à des variations marquées de la teneur en $\beta$-carotène des fromages au lait de vache en période de pâturage $\left(3,70,4,26\right.$ et $5,65 \mathrm{mg} \cdot \mathrm{kg}^{-1} \mathrm{e}$ de MG pour les races Abondance, Holstein et Montbéliarde, respectivement). 
Tableau 3. Caractéristiques nutritionnelles du fromage au lait de vache en fonction de la nature de la ration fourragère ${ }^{1}$ (Lucas et al 2006a).

\begin{tabular}{|c|c|c|c|c|c|c|c|c|c|c|c|}
\hline Nature de la ration fourragère $^{2}$ & PA1 & PP1 & PP2 & PP1/F & PP2/F & PT/F & Ferm & FP1 & FP2 & FT & Maïs \\
\hline Nombre de fromages & 23 & 15 & 31 & 32 & 91 & 17 & 12 & 36 & 32 & 8 & 9 \\
\hline \multicolumn{12}{|l|}{ Complémentation } \\
\hline Concentrés ( $\%$ de la ration) & 13 & 19 & 20 & 23 & 26 & 29 & 31 & 32 & 32 & 40 & 25 \\
\hline Vitamine A $\left(10^{3} \times\right.$ Ul.vache $\left.{ }^{-1} . j^{-1}\right)$ & 19 & 25 & 36 & 67 & 55 & 87 & 80 & 55 & 102 & 79 & 145 \\
\hline Vitamine E (UI.vache $\left.{ }^{-1} \cdot j^{-1}\right)$ & 32 & 60 & 63 & 115 & 96 & 211 & 51 & 102 & 129 & 167 & 187 \\
\hline \multicolumn{12}{|l|}{ Race (\% du troupeau) } \\
\hline Abondance & 74 & 38 & 59 & 49 & 44 & 15 & 50 & 66 & 43 & 39 & 22 \\
\hline Holstein & 0 & 19 & 7 & 5 & 13 & 47 & 21 & 7 & 8 & 14 & 22 \\
\hline Montbéliarde & 17 & 32 & 30 & 40 & 34 & 37 & 17 & 26 & 43 & 47 & 36 \\
\hline Stade de lactation (mois) & 7.3 & 4.9 & 5.9 & 6.0 & 5.8 & 5.8 & 4.9 & 4.5 & 5.0 & 4.9 & 5.3 \\
\hline Niveau de production (L.vache $\left.{ }^{-1} \cdot j^{-1}\right)$ & 14 & 20 & 16 & 20 & 17 & 20 & 18 & 18 & 18 & 22 & 21 \\
\hline \multicolumn{12}{|l|}{ Acides gras ( $\%$ des AG totaux) ${ }^{3}$} \\
\hline Saturés & $64^{\mathrm{a}}$ & $67^{b}$ & $68^{\mathrm{bc}}$ & $67^{\mathrm{b}}$ & $69^{c}$ & $69^{\mathrm{C}}$ & $72^{d}$ & $72^{\mathrm{d}}$ & $72^{d}$ & $73^{d}$ & $74^{\mathrm{d}}$ \\
\hline $\mathrm{C} 4: 0$ & $4,2^{a b}$ & $4,0^{a}$ & $4,2^{b}$ & $4,2^{\mathrm{ab}}$ & $4,4^{\mathrm{c}}$ & $4,4^{\mathrm{bc}}$ & $4,1^{\mathrm{ab}}$ & $4,1^{\mathrm{a}}$ & $3,9^{a}$ & $4,1^{\mathrm{ab}}$ & $4,3^{\mathrm{bc}}$ \\
\hline C6:0-C10:0 & $6,2^{a}$ & $8,3^{d}$ & $7,6^{\mathrm{b}}$ & $8,0^{b c}$ & $7,9^{b c}$ & $7,9^{\mathrm{bc}}$ & $8,2^{\mathrm{cd}}$ & $8,1^{\text {cd }}$ & $8,4^{d}$ & $8,8^{d}$ & $8,5^{\mathrm{d}}$ \\
\hline C12:0-C16:0 & $40^{a}$ & $42^{b}$ & $43^{\mathrm{bc}}$ & $42^{b}$ & $44^{c}$ & $44^{\mathrm{c}}$ & $47^{d}$ & $48^{d}$ & $48^{d}$ & $48^{d}$ & $49^{d}$ \\
\hline C18:0 & $9,2^{d}$ & $8,8^{\mathrm{cd}}$ & $8,9^{c d}$ & $9,2^{d}$ & $8,5^{\mathrm{bc}}$ & $8,1^{\mathrm{ab}}$ & $8,7^{\mathrm{cd}}$ & $7,4^{\mathrm{a}}$ & $7,7^{\mathrm{a}}$ & $7,9^{\mathrm{ab}}$ & $8,4^{\mathrm{bc}}$ \\
\hline Monoinsaturés & $29^{d}$ & $27^{c}$ & $27^{c}$ & $27^{\mathrm{c}}$ & $26^{b}$ & $26^{b}$ & $24^{a}$ & $23^{a}$ & $23^{a}$ & $23^{a}$ & $23^{a}$ \\
\hline C18:1 c9 (n-9) & $20^{d}$ & $18^{\mathrm{b}}$ & $19^{c}$ & $18^{\mathrm{C}}$ & $18^{\mathrm{b}}$ & $17^{\mathrm{ab}}$ & $17^{\mathrm{ab}}$ & $16^{\mathrm{a}}$ & $16^{a}$ & $16^{\mathrm{a}}$ & $16^{\mathrm{a}}$ \\
\hline C18:1 $t 11(+t 10)$ & $3,1^{\mathrm{e}}$ & $3,7^{f}$ & $2,5^{\mathrm{c}}$ & $2,8^{d}$ & $2,4^{\mathrm{C}}$ & $2,5^{\mathrm{cd}}$ & $1,3^{b}$ & $1,4^{\mathrm{b}}$ & $1,4^{\mathrm{b}}$ & $1,4^{\mathrm{b}}$ & $1,0^{a}$ \\
\hline Polyinsaturés & $5,2^{\mathrm{e}}$ & $4,4^{d}$ & $4,1^{\mathrm{c}}$ & $4,4^{d}$ & $4,1^{\mathrm{c}}$ & $4,3^{d}$ & $3,2^{\mathrm{ab}}$ & $3,5^{\mathrm{b}}$ & $3,7^{\mathrm{b}}$ & $3,7^{\mathrm{bc}}$ & $2,9^{a}$ \\
\hline C18:2 c9c12 (n-6) & $1,7^{\mathrm{c}}$ & $1,3^{a}$ & $1,5^{\mathrm{b}}$ & $1,5^{\mathrm{b}}$ & $1,5^{\mathrm{b}}$ & $1,5^{\mathrm{b}}$ & $1,4^{\mathrm{ab}}$ & $1,5^{\mathrm{b}}$ & $1,6^{b}$ & $1,6^{\mathrm{bc}}$ & $1,4^{\mathrm{ab}}$ \\
\hline C18:2 c9t11 (CLA) ${ }^{4}$ & $1,3^{\mathrm{e}}$ & $1,5^{\mathrm{e}}$ & $1,1^{\mathrm{cd}}$ & $1,2^{d}$ & $1,0^{\mathrm{C}}$ & $1,0^{\mathrm{cd}}$ & $0,52^{\mathrm{ab}}$ & $0,63^{b}$ & $0,61^{b}$ & $0,62^{b}$ & $0,43^{a}$ \\
\hline C18:3 c9c12c15 (n-3) & $1,1^{\mathrm{d}}$ & $0,79^{b c}$ & $0,71^{b}$ & $0,8^{\mathrm{C}}$ & $0,77^{\mathrm{bc}}$ & $0,82^{c}$ & $0,68^{b}$ & $0,73^{b}$ & $0,81^{c}$ & $0,76^{\mathrm{bc}}$ & $0,49^{a}$ \\
\hline Trans (CLA non inclus) & $5,7^{d}$ & $6,0^{\mathrm{d}}$ & $4,4^{b}$ & $5,0^{\mathrm{C}}$ & $4,4^{b}$ & $4,6^{b c}$ & $2,9^{a}$ & $2,9^{a}$ & $2,9^{a}$ & $3,1^{a}$ & $2,7^{\mathrm{a}}$ \\
\hline \multicolumn{12}{|l|}{ Vitamines et caroténoïdes ${ }^{5}$} \\
\hline$\beta$-Carotène (mg. $\left.\mathrm{kg}^{-1} \mathrm{MG}\right)$ & $4,3^{\mathrm{C}}$ & $5,2^{\mathrm{e}}$ & $4,7^{\mathrm{cd}}$ & $4,1^{\mathrm{c}}$ & $4,9^{d}$ & $4,4^{\mathrm{cd}}$ & $2,3^{b}$ & $1,8^{a b}$ & $1,8^{\mathrm{ab}}$ & $1,4^{\mathrm{a}}$ & $1,2^{\mathrm{a}}$ \\
\hline Xanthophylles (mg.kg-1 MG) & $0,33^{d}$ & $0,41^{d}$ & $0,31^{\mathrm{c}}$ & $0,30^{\mathrm{C}}$ & $0,26^{\mathrm{b}}$ & $0,28^{\mathrm{bc}}$ & $0,16^{a}$ & $0,24^{b}$ & $0,24^{b}$ & $0,23^{a b}$ & $0,15^{\mathrm{a}}$ \\
\hline Vitamine A (mg.kg $\left.{ }^{-1} \mathrm{MG}\right)$ & $5,1^{\mathrm{bc}}$ & $5,9^{\mathrm{cd}}$ & $5,8^{\mathrm{cd}}$ & $6,0^{\mathrm{d}}$ & $5,3^{\mathrm{c}}$ & $6,1^{\mathrm{d}}$ & $5,1^{\text {bc }}$ & $4,8^{\mathrm{ab}}$ & $4,7^{\mathrm{ab}}$ & $4,3^{\mathrm{ab}}$ & $3,8^{a}$ \\
\hline Vitamine $\mathrm{E}$ (mg. $\left.\mathrm{kg}^{-1} \mathrm{MG}\right)$ & $7,7^{\mathrm{bc}}$ & $8,1^{\mathrm{c}}$ & $7,9^{c}$ & $6,9^{b}$ & $7,1^{\mathrm{b}}$ & $7,6^{\text {bc }}$ & $3,5^{\mathrm{a}}$ & $3,2^{a}$ & $3,5^{\mathrm{a}}$ & $3,0^{a}$ & $4,1^{\mathrm{a}}$ \\
\hline Pot. antiox. (mol Fe $\left.{ }^{2+} . \mathrm{kg}^{-1} \mathrm{MS}\right)^{6}$ & $13^{\mathrm{C}}$ & $15^{\mathrm{c}}$ & $15^{\mathrm{C}}$ & $14^{\mathrm{C}}$ & $15^{\mathrm{c}}$ & $14^{\mathrm{C}}$ & $13^{\mathrm{bc}}$ & $11^{\mathrm{ab}}$ & $11^{\mathrm{a}}$ & $11^{\mathrm{a}}$ & $11^{\mathrm{ab}}$ \\
\hline
\end{tabular}

1 Moyennes ajustées pour les autres facteurs de production. 2 PA1 : pâturage sur une prairie d'alpage en première utilisation. PP1 : pâturage sur une prairie permanente en première utilisation. PP2 : pâturage sur une prairie permanente en seconde utilisation. PP1/F : pâturage sur une prairie permanente en première utilisation avec une complémentation en foin. PP2/F : pâturage sur une prairie permanente en seconde utilisation avec une complémentation en foin. PT/F : pâturage sur une prairie temporaire avec une complémentation en foin. Ferm : riche en herbe fermentée (ensilée ou enrubannée). FP1 : foin de prairie permanente. FP2 : riche en regain de praire permanente. FT : riche en foin de prairie temporaire. Maïs : riche en ensilage de maïs. ${ }^{3}$ AG : acides gras. ${ }^{4}$ CLA : acide linoléique conjugué. ${ }^{5}$ MG : matières grasses. ${ }^{6}$ Pot. antiox. : potentiel antioxydant. MS : matières sèches. $a, b, c, d, e$ Les valeurs ne présentant aucune lettre en commun diffèrent significativement $(P<0,05)$.

Figure 2. Relations entre le niveau de supplémentation vitaminique quotidienne par vache des rations à base de pâturage $(\square)$ et des rations à base de fourrages conservés ( $\square$ ) et les teneurs en vitamine $A$ et $E$ du fromage. Les résultats sont exprimés en $m g . \mathrm{kg}^{-1}$ de matières grasses (Lucas et al 2006a).

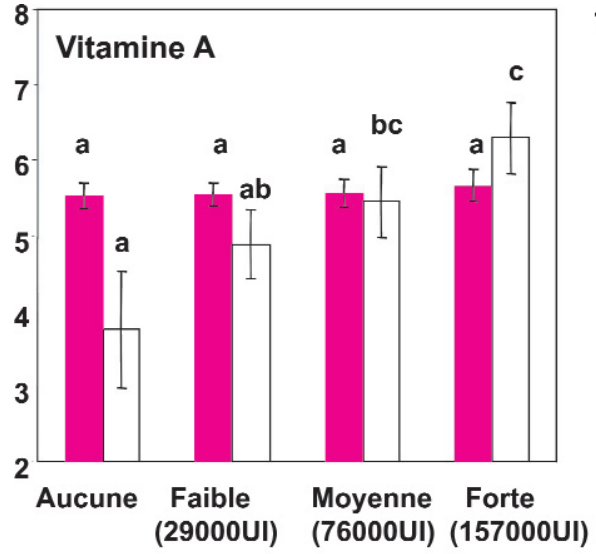

Supplémentation en vitamine A

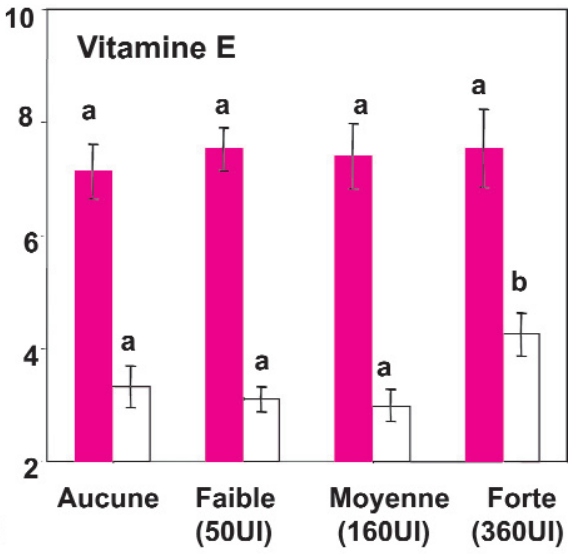

Supplémentation en vitamine E

\section{5 / Relations entre les condi- tions de production du lait et le profil en acide gras du fromage (Lucas 2005, Lucas et al 2006a)}

Cette étude a montré que l'espèce animale (vache vs chèvre) est un facteur de variation important du profil en $\mathrm{AG} d u$ fromage (tableau 2), confirmant les données disponibles sur le lait (Chilliard et Lamberet 2001). Ces différences concernent plus particulièrement le C8:0 et le C10:0, en proportions 2 et 3 fois plus importantes dans la $\mathrm{MG}$ du fromage au lait de chèvre, et le $\mathrm{C} 4: 0$, présent en proportion plus importante dans celle du fromage au lait de vache. D'autre part, la MG du 
Tableau 4. Caractéristiques nutritionnelles du fromage au lait de chèvre en fonction de la nature de la ration fourragère ${ }^{1}$ (Lucas et al 2005).

\begin{tabular}{|c|c|c|c|c|c|c|c|}
\hline Nature de la ration fourragère ${ }^{2}$ & $P P>F$ & $P L>F$ & $P=F$ & $\mathrm{P}<\mathrm{F}$ & FP & FL & FT \\
\hline Nombre de fromages & 9 & 8 & 20 & 13 & 13 & 35 & 28 \\
\hline Concentrés ( $\%$ de la ration) & 24 & 36 & 31 & 36 & 42 & 38 & 42 \\
\hline \multicolumn{8}{|l|}{ Complémentation en vitamines } \\
\hline Vitamine $A\left(10^{3} \times\right.$ Ul.chèvre $\left.{ }^{-1} \cdot j^{-1}\right)$ & 1,2 & 16 & 4,0 & 2,0 & 3,6 & 6,5 & 10 \\
\hline Vitamine $E\left(U I . c h e ̀ v r e^{-1} \cdot j^{-1}\right)$ & 0,8 & 40 & 10 & 5,5 & 4,3 & 9,2 & 20 \\
\hline \multicolumn{8}{|l|}{ Race (\% du troupeau) } \\
\hline Alpine & 80 & 97 & 77 & 73 & 84 & 67 & 55 \\
\hline Saanen & 20 & 3 & 23 & 27 & 16 & 33 & 45 \\
\hline Stade de lactation (mois) & 6,0 & 5,6 & 6,4 & 5,3 & 5,2 & 5,2 & 5,8 \\
\hline $\begin{array}{l}\text { Niveau de production } \\
\left(\text { L.chèvre }{ }^{-1} . j^{-1}\right)\end{array}$ & 1,7 & 2,1 & 2,1 & 2,4 & 2,0 & 2,1 & 2,3 \\
\hline Xanthophylles (mg. $\left.\mathrm{kg}^{-1} \mathrm{MG}\right)^{3}$ & $0,22^{b}$ & $0,20^{b}$ & $0,16^{\mathrm{ab}}$ & $0,15^{\mathrm{ab}}$ & $0,18^{\mathrm{b}}$ & $0,13^{a}$ & $0,19^{b}$ \\
\hline Vitamine A (mg.kg $\left.{ }^{-1} \mathrm{MG}\right)^{3}$ & $8,2^{b}$ & $8,7^{\mathrm{b}}$ & $6,7^{a}$ & $6,3^{a}$ & $5,4^{a}$ & $6,5^{a}$ & $5,9^{a}$ \\
\hline Vitamine $\mathrm{E}\left(\mathrm{mg} \cdot \mathrm{kg}^{-1} \mathrm{MG}\right)^{3}$ & $9,4^{d}$ & $8,4^{\text {cd }}$ & $7,7^{\mathrm{bc}}$ & $7,2^{b}$ & $5,6^{\mathrm{a}}$ & $5,2^{a}$ & $5,6^{\mathrm{a}}$ \\
\hline Pot. antiox. (mol Fe $\left.{ }^{2+} \cdot \mathrm{kg}^{-1} \mathrm{MS}\right)^{4}$ & $16^{\mathrm{C}}$ & $15^{\mathrm{c}}$ & $12^{\mathrm{b}}$ & $11^{\mathrm{ab}}$ & $12^{\mathrm{ab}}$ & $10^{a}$ & $10^{\mathrm{a}}$ \\
\hline
\end{tabular}

1 Moyennes ajustées pour les autres facteurs de production. $2 \mathrm{PP}>\mathrm{F}$ : pâturage de prairie permanente en majorité avec une faible complémentation en foin. $\mathrm{PL}>\mathrm{F}$ : pâturage de prairie de luzerne en majorité avec une faible complémentation en foin. $\mathrm{P}=\mathrm{F}$ : proportions équivalentes de pâturage et de foin. $P<F$ : faible proportion de pâturage avec une forte complémentation en foin. $F P$ : foin de prairie permanente. FL : foin de luzerne. $\mathrm{FT}$ : foin de prairie temporaire. ${ }^{3} \mathrm{MG}$ : matières grasses. ${ }^{4}$ Pot. antiox. : potentiel antioxydant. MS : matières sèches. $a, b, c, d$ : Les valeurs ne présentant aucune lettre en commun diffèrent significativement $(P<0,05)$.

fromage au lait de chèvre est également la plus riche en $\mathrm{C} 18: 2 \mathrm{n}-6$, tandis que l'inverse est observé pour le C18:1 trans-11 et le C18:2 cis-9, trans-11 (CLA) présents en quantités plus importantes dans le fromage au lait de vache. Concernant ces 3 derniers AG, la variabilité intra-espèces est cependant supérieure à la variabilité inter espèce.

Dans la gamme de conditions de production du lait étudiées, le profil en AG du fromage au lait de vache varie principalement avec la nature de la ration fourragère et plus particulièrement le mode de conservation des fourrages (pâturage vs fourrages conservés). Lorsque les animaux consomment des rations à base de pâturage, les fromages sont plus riches en AG saturés et insaturés à longue chaîne $(\geq \mathrm{C} 18)$ et plus pauvres en $\mathrm{AG}$ saturés à courte et moyenne chaîne (C6:0 à C16:0) (tableau 3). Ces résultats s'expliquent par la plus grande richesse de l'herbe verte en $\mathrm{AG}$ et plus particulièrement en C18:3 comparée aux fourrages conservés en raison d'une dégradation oxydative de ces composés au cours de la récolte et du stockage des fourrages conservés (Boufaïed et al 2003). Pour la même raison, la complémentation en foin des rations à base de pâturage conduit à de plus faibles proportions de CLA et de C18:1 trans-11 dans le fromage (tableau 3).
En période de pâturage, le stade de maturité de l'herbe est également un facteur de variation du profil en $\mathrm{AG}$ du fromage au lait de vache. Ainsi, les fromages ont présenté des proportions plus importantes de CLA et de C18:1 trans-11 et une proportion plus faible de C16:0 lorsque les animaux, recevant une ration fourragère à base exclusivement d'herbe verte, pâturaient une prairie permanente en première utilisation comparée à une prairie permanente de rang d'utilisation supérieure (tableau 3). Ces résultats peuvent en partie s'expliquer par la diminution de la teneur en AG totaux, et notamment en C18:3, de l'herbe au cours de son développement (Boufaïed et al 2003).

Ce travail a également confirmé que les fromages au lait de vache produits en alpage se caractérisent par un profil en AG particulier (Bugaud et al 2001, Collomb et al 2001). Ce sont notamment les plus riches en C18:1 cis-9, C18:2 n-6 et C18:3 n-3 et les plus pauvres en $\mathrm{AG}$ saturés à courte et moyenne chaîne (C6:0 à C14:0) (tableau 3). Un apport alimentaire plus élevé en AG à longue chaîne lié à la composition botanique particulière des prairies d'alpage (Bugaud et al 2000), une plus forte mobilisation des réserves corporelles en $A G$ en raison des conditions plus difficiles de pâturage en alpage (Bossart et al 1985, Chilliard et al
2000) et/ou une inhibition de la biohydrogénation ruminale par des composés alimentaires spécifiques des prairies d'alpage tels que des polyphénols ou des terpènes (Leiber et al 2005) sont les principales hypothèses pouvant expliquer ces résultats.

Par ailleurs, lorsque les vaches consomment une ration à base de fourrages conservés, le profil en AG du fromage au lait de vache varie en fonction de la nature des fourrages. Le fromage est notamment moins riche en CLA, en C18:1 trans-11 et en C18:3 n-3 lorsque les vaches consomment une ration riche en ensilage de maïs comparée à une ration à base de foin ou d'herbe fermentée (tableau 3).

Le profil en AG du fromage au lait de vache dépend également du niveau de complémentation en concentrés des rations, mais uniquement lorsque celles-ci sont à base d'herbe verte. Ces variations concernent plus particulièrement le CLA et le C18:1 trans-11, présents en quantités d'autant plus faibles dans le fromage que le niveau de complémentation en concentrés est important. Par exemple, les teneurs du fromage en CLA et en C18:1 trans-11 étaient respectivement égales à 1,4 et 3,2\% des AG totaux avec une proportion de concentrés dans la ration de l'ordre de $15 \%$ contre 1,1 et $2,5 \%$ des AG totaux avec un niveau de concentrés représentant $41 \%$ de la ration. Ces résultats, qui confirment de précédents travaux réalisés sur le lait (Stockdale et al 2003), reflètent une plus faible quantité d'herbe verte consommée par les vaches lorsque la quantité de concentrés dans la ration augmente et donc un plus faible niveau de consommation en C18:3, généralement plus abondant dans l'herbe verte que dans les concentrés (Morand-Fehr et Tran 2001).

Contrairement au profil en $\mathrm{AG}$ du fromage au lait de vache, celui du fromage au lait de chèvre n'a pas été, dans cette étude, fortement influencé par la nature de la ration fourragère. Les variations du profil en $\mathrm{AG}$ de ce fromage ont été principalement liées à la proportion de MG apportées par les concentrés dans la ration, à la présence ou non de lin dans les concentrés et au stade de lactation des animaux (tableau 5). Ce fromage est ainsi plus riche en $\mathrm{AG}$ à longue chaîne $(\geq \mathrm{C} 18)$ et plus pauvre en $\mathrm{AG}$ saturés à courte et moyenne chaîne (C6:0 à C16:0) lorsque la proportion de $\mathrm{MG}$ apportées par les concentrés dans la ration est plus importante, les concentrés contien- 
Tableau 5. Profil en acides gras (\% des acides gras totaux) du fromage au lait de chèvre en fonction de la proportion de matières grasses apportées par les concentrés dans la ration, de la composition en lin des concentrés et du stade de lactation moyen du troupeau ${ }^{1}$ (Lucas et al 2005).

\begin{tabular}{|c|c|c|c|c|c|c|c|c|}
\hline & \multicolumn{3}{|c|}{$\begin{array}{l}\text { MG apportées } \\
\text { par les concentrés }\end{array}$} & \multicolumn{2}{|c|}{$\begin{array}{c}\text { Lin } \\
\text { dans les concentrés }\end{array}$} & \multicolumn{3}{|c|}{$\begin{array}{l}\text { Stade de lactation } \\
\text { du troupeau }\end{array}$} \\
\hline & $0,7 \%$ & $1,3 \%$ & $2,1 \%$ & Présence & Absence & 2,9 mois & 5,3 mois & 7,6 mois \\
\hline Nombre de fromages & 41 & 48 & 37 & 48 & 78 & 25 & 59 & 42 \\
\hline \multicolumn{9}{|l|}{$\begin{array}{l}\text { Nature de la ration fourragère }(\% \\
\text { des fromages) }\end{array}$} \\
\hline Pâturage & 46 & 38 & 35 & 48 & 35 & 20 & 46 & 43 \\
\hline Fourrages conservés & 54 & 63 & 65 & 52 & 65 & 80 & 54 & 57 \\
\hline \multicolumn{9}{|l|}{ Complémentation en concentrés } \\
\hline $\begin{array}{l}\text { Niveau de complémentation (\% de } \\
\text { la ration) }\end{array}$ & 28 & 38 & 45 & 41 & 34 & 37 & 37 & 37 \\
\hline $\begin{array}{l}\text { Matières grasses apportées (\% de } \\
\text { la ration) }\end{array}$ & - & - & - & 1,9 & 1,0 & 1,3 & 1,3 & 1,4 \\
\hline Présence de lin (\% des fromages) & 0 & 35 & 84 & - & - & 32 & 37 & 43 \\
\hline \multicolumn{9}{|l|}{ Race $(\%$ du troupeau) } \\
\hline Alpine & 71 & 76 & 65 & 64 & 75 & 71 & 68 & 77 \\
\hline Saanen & 29 & 24 & 35 & 36 & 25 & 29 & 32 & 23 \\
\hline Stade de lactation (mois) & 5,4 & 5,6 & 5,7 & 5,7 & 5,5 & - & - & - \\
\hline Niveau de production (L.chèvre ${ }^{-1} . j^{-1}$ ) & 1,9 & 2,3 & 2,3 & 2,4 & 2,0 & 2,4 & 2,3 & 1,8 \\
\hline Acides gras saturés & 74 & 73 & 71 & $71^{\mathrm{a}}$ & $73^{b}$ & $71^{\mathrm{a}}$ & $74^{\mathrm{b}}$ & $74^{\mathrm{b}}$ \\
\hline $\mathrm{C} 4: 0$ & 2,8 & 2,8 & 2,8 & 2,8 & 2,7 & $2,6^{\mathrm{a}}$ & $2,9^{b}$ & $2,7^{b}$ \\
\hline C6:0-C10:0 & 17 & 17 & 16 & 17 & 16 & $16^{\mathrm{a}}$ & $17^{b}$ & $18^{\mathrm{b}}$ \\
\hline C12:0-C16:0 & 38 & 40 & 38 & $37^{a}$ & $41^{\mathrm{b}}$ & $37^{\mathrm{a}}$ & $39^{b}$ & $41^{\mathrm{c}}$ \\
\hline C18:0 & 11 & 10 & 11 & $11^{\mathrm{b}}$ & $9,9^{\mathrm{a}}$ & $11^{\mathrm{c}}$ & $11^{\mathrm{b}}$ & $9,7^{\mathrm{a}}$ \\
\hline Acides gras monoinsaturés & $21^{\mathrm{a}}$ & $22^{\mathrm{a}}$ & $23^{b}$ & 22 & 22 & $25^{\mathrm{b}}$ & $22^{a}$ & $21^{a}$ \\
\hline C18:1 c9 (n-9) & $17^{\mathrm{a}}$ & $18^{\mathrm{ab}}$ & $18^{\mathrm{b}}$ & 17 & 17 & $20^{\mathrm{b}}$ & $17^{\mathrm{a}}$ & $16^{\mathrm{a}}$ \\
\hline$C 18: 1 t 11+t 10)$ & $0,88^{\mathrm{a}}$ & $1,0^{\mathrm{a}}$ & $1,4^{b}$ & 1,1 & 1,1 & 1,1 & 1,1 & 1,1 \\
\hline Acides gras polyinsaturés & $4,3^{\mathrm{ab}}$ & $4,1^{\mathrm{a}}$ & $4,5^{\mathrm{b}}$ & $4,7^{b}$ & $3,9^{a}$ & $4,5^{\mathrm{b}}$ & $4,2^{\mathrm{a}}$ & $4,1^{\mathrm{a}}$ \\
\hline $\mathrm{C} 18: 2$ c9c12 (n-6) & $2,1^{\mathrm{a}}$ & $2,2^{b}$ & $2,4^{\mathrm{C}}$ & 2,3 & 2,2 & $2,4^{b}$ & $2,2^{\mathrm{a}}$ & $2,1^{\mathrm{a}}$ \\
\hline $\mathrm{C} 18: 2$ c9t11 (CLA) ${ }^{3}$ & $0,32^{\mathrm{a}}$ & $0,38^{\mathrm{b}}$ & $0,50^{\mathrm{c}}$ & 0,40 & 0,40 & $0,35^{\mathrm{a}}$ & $0,40^{b}$ & $0,45^{\mathrm{c}}$ \\
\hline $\mathrm{C} 18: 3 c 9 c 12 c 15(\mathrm{n}-3)$ & $0,92^{\mathrm{b}}$ & $0,71^{a}$ & $0,72^{\mathrm{a}}$ & $1,0^{b}$ & $0,62^{\mathrm{a}}$ & 0,78 & 0,78 & 0,76 \\
\hline Acides gras trans (CLA non inclus) & $2,2^{\mathrm{a}}$ & $2,3^{\mathrm{a}}$ & $2,9^{\mathrm{b}}$ & $2,7^{b}$ & $2,2^{\mathrm{a}}$ & 2,6 & 2,5 & 2,4 \\
\hline
\end{tabular}

${ }^{1}$ Moyennes ajustées pour les autres facteurs de production. ${ }^{2}$ MG : matières grasses. ${ }^{3} \mathrm{CLA}$ : acide linoléique conjugué. a,b,c Les valeurs ne présentant aucune lettre en commun diffèrent significativement $(P<0,05)$.

nent du lin et les animaux sont en début de lactation. L'aptitude des compléments lipidiques à influencer le profil en $A G$ du lait de chèvre (Chilliard et al 2003) a donc été confirmée en conditions réelles de production. L'influence de la présence de lin dans les concentrés est en grande partie due à sa richesse en C18:3. L'effet du stade de lactation s'explique quant à lui par un déficit énergétique des animaux en début de lactation à l'origine d'une mobilisation des réserves corporelles en $\mathrm{AG}$, notamment en C18:1 cis-9 et dans une moindre mesure en C18:0, et d'une sécrétion de ces AG dans le lait (Chilliard et al 2003).

Les différences de facteurs de variation du profil en $A G$ du fromage entre les vaches et les chèvres s'expliquent davantage par des différences de pratiques d'élevage entre ces deux espèces animales que par un effet direct de l'espèce animale. Dans cette étude, les proportions de concentrés et de MG apportées par les concentrés dans la ration étaient ainsi plus élevées chez les chèvres $(36 \%$ et $1,3 \%$, respectivement) que chez les vaches (26\% et $0,65 \%$, respectivement). D'autre part, la présence de lin dans les concentrés était rare chez les vaches, tandis que la répartition des mises bas était beaucoup moins dispersée chez les chèvres ce qui a conduit à une expression plus marquée du stade de lactation du troupeau chez cette espèce. Par conséquent, l'effet de la nature de la ration fourragère a probablement été masqué chez les chèvres par ceux de la nature des concentrés et du stade de lactation.

\section{6 / Typologies des fromages (Lucas 2005)}

L'étude de la relation entre chaque facteur de production et la teneur dans le fromage en chacun des composés d'intérêt nutritionnel étudiés a mis en évidence que certains facteurs de production influencent plusieurs familles de composés. Selon les conditions de production du lait, le fromage est donc susceptible de présenter un profil nutritionnel différent. Pour illustrer cela, nous avons réalisé des typologies de fromages, une chez les vaches et une autre chez les chèvres, sur la base de leurs caractéristiques nutritionnelles (figures 3 et 4 , tableaux 6 et 7). Par ailleurs, en conditions réelles de production, chaque pratique d'élevage est 


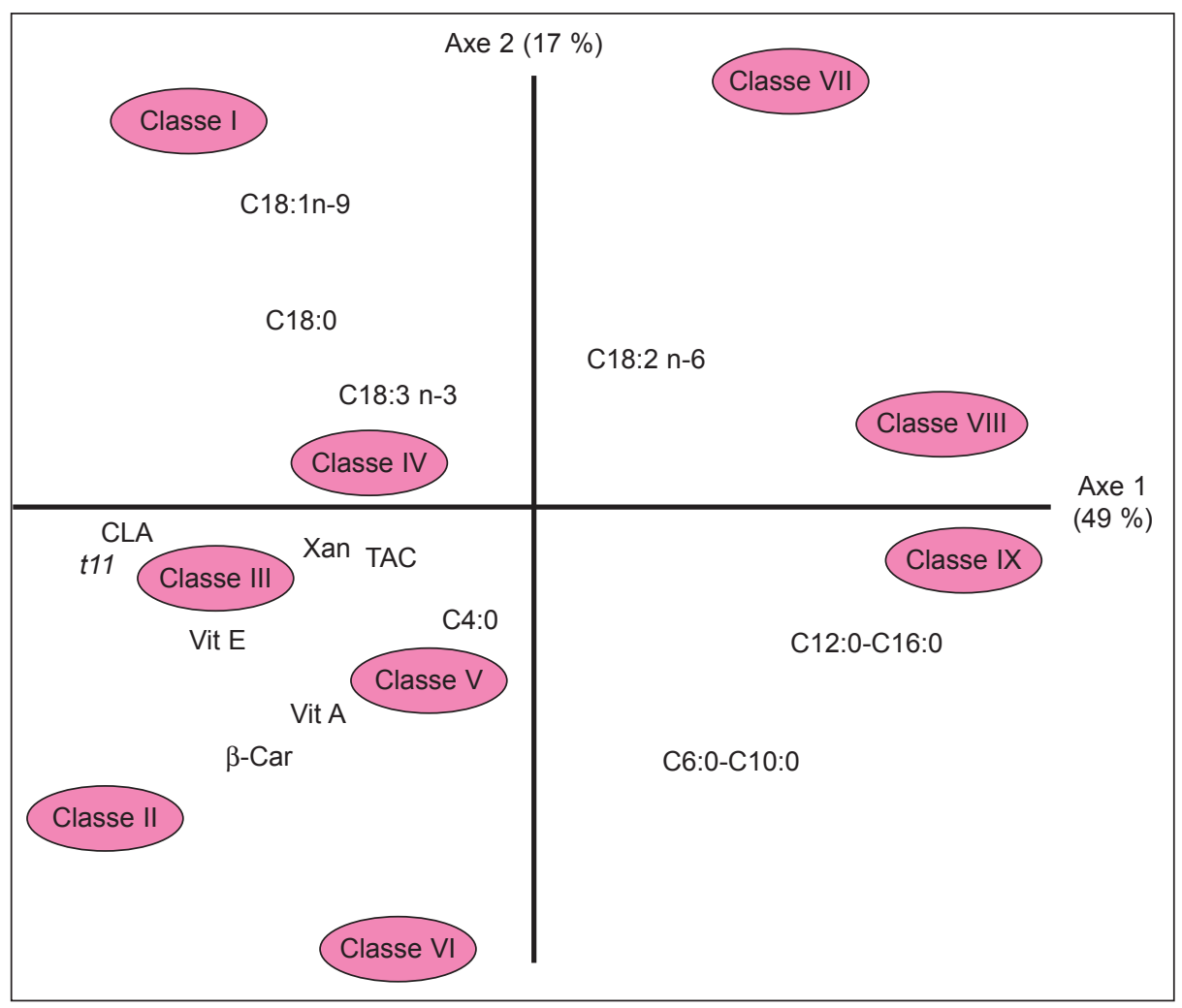

Figure 3. Représentation graphique de la typologie des fromages au lait de vache sur le plan factoriel représenté par les 2 premiers axes d'une analyse factorielle des correspondances multiples réalisée sur 6 caractéristiques du fromage (C12:0C16:0, C18:1 n-9, CLA, vitamine A, vitamine $E$ et $\beta$-carotène). Les caractéristiques nutritionnelles moyennes des classes de fromages et les conditions de production du lait associées sont présentées dans le tableau 6. $\beta$-Car: $\beta$-carotè$n e$. Vit $A$ : vitamine $A$. Vit $E$ : vitamine $E$. Xan : xanthophylles. TAC : potentiel antioxydant. t11 : C18:1 t11. CLA : C18:2 c9t11. C6:0-C10:0:C6:0+C8:0+C10:0. C12:0-C16:0:C12:0+C14:0+C16:0 (Lucas 2005).

Figure 4. Représentation graphique de la typologie des fromages au lait de chèvre sur le plan factoriel représenté par les 2 premiers axes d'une analyse factorielle des correspondances multiples réalisée sur 5 caractéristiques du fromage (C12:0C16:0, C18:1 n-9, C18:3 n-3, vitamine A et vitamine E). Les caractéristiques nutritionnelles moyennes des classes de fromages et les conditions de production du lait associées sont présentées dans le tableau 7. Vit $A$ : vitamine $A$. Vit $E$ : vitamine E. Xan : xanthophylles. TAC : potentiel antioxydant. $t 11$ : C18:1 t11. CLA : C18:2 c9t11. C6:0-C10:0:C6:0+C8:0+ C10:0. C12:0-C16:0:C12:0+C14:0+ C16:0 (Lucas 2005).

intégrée au sein d'un système de production cohérent. Il existe donc de nombreuses associations plus ou moins fortes entre pratiques d'élevage. Ces dernières pouvant agir de façon synergique ou antagoniste sur les caractéristiques nutritionnelles du fromage, la composition du fromage varie donc avec des ensembles de pratiques d'élevage (tableaux 6 et 7 ).

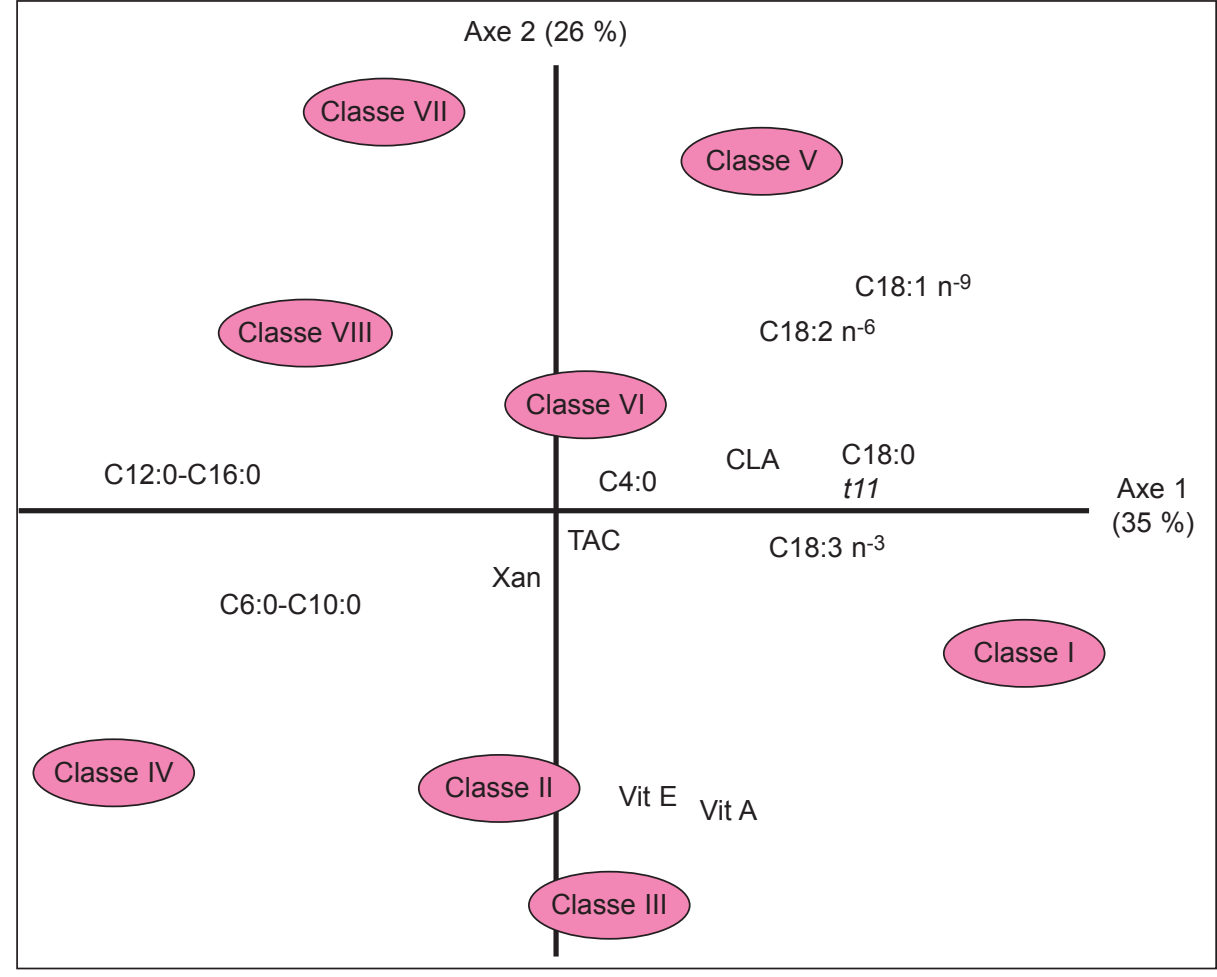

Nous avons constitué 9 groupes de fromages au lait de vaches. Ces groupes de fromages se différencient à la fois par leur profil en AG et par leurs teneurs en micronutriments liposolubles (figure 3). Cette typologie met en évidence l'existence d'une forte association entre ces deux familles de composés. Le profil en AG et les teneurs en micronutriments liposolubles du fro- mage varient ainsi simultanément dans un sens a priori favorable pour la santé. En effet, les fromages les plus riches en micronutriments liposolubles sont aussi les plus riches en $A G$ à longue chaîne $(\geq \mathrm{C} 18)$ et donc les moins riches en $\mathrm{AG}$ saturés à courte et moyenne chaîne (C6:0 à C16:0). Ces groupes sont associés à des ensembles de pratique d'élevage (tableau 6) et en particulier à la 
Tableau 6. Typologie des fromages au lait de vache: caractéristiques nutritionnelles moyennes des classes de fromages présentées dans la figure 3 et conditions de production du lait associées (Lucas 2005).

\begin{tabular}{|c|c|c|c|c|c|c|c|c|c|}
\hline Classes de fromages & $\mathbf{I}$ & II & III & IV & $\mathbf{V}$ & VI & VII & VIII & IX \\
\hline Nombre de fromages & 21 & 14 & 73 & 51 & 22 & 22 & 13 & 42 & 48 \\
\hline \multicolumn{10}{|l|}{ Ration fourragère $(\%)^{1}$} \\
\hline Pâturage & $86^{\mathrm{e}}$ & $83^{e}$ & $75^{\mathrm{d}}$ & $52^{c}$ & $36^{b}$ & $57^{\mathrm{c}}$ & $2^{a}$ & $0^{a}$ & $0^{a}$ \\
\hline Alpage & $86^{d}$ & $14^{\mathrm{C}}$ & $0^{a}$ & $4^{\mathrm{ab}}$ & $0^{\mathrm{a}}$ & $8^{b c}$ & $0^{a}$ & $0^{a}$ & $0^{a}$ \\
\hline Prairie permanente & $0^{\mathrm{a}}$ & $68^{d}$ & $71^{d}$ & $47^{c}$ & $23^{b}$ & $30^{b}$ & $2^{a}$ & $0^{a}$ & $0^{a}$ \\
\hline Prairie temporaire & $0^{a}$ & $2^{a}$ & $4^{\mathrm{a}}$ & $0^{\mathrm{a}}$ & $13^{b}$ & $19^{b}$ & $0^{a}$ & $0^{\mathrm{a}}$ & $0^{\mathrm{a}}$ \\
\hline $1^{\mathrm{e}}$ utilisation & $76^{\mathrm{e}}$ & $41^{d}$ & $25^{\mathrm{c}}$ & $17^{\mathrm{bc}}$ & $9^{\mathrm{ab}}$ & $9^{a b}$ & $0^{\mathrm{a}}$ & $0^{\mathrm{a}}$ & $0^{\mathrm{a}}$ \\
\hline$\geq 2^{\mathrm{e}}$ utilisation & $10^{a}$ & $43^{c}$ & $49^{c}$ & $35^{\mathrm{bc}}$ & $27^{\mathrm{b}}$ & $48^{c}$ & $2^{a}$ & $0^{a}$ & $0^{a}$ \\
\hline Foin & $0^{\mathrm{a}}$ & $2^{a}$ & $5^{\mathrm{ab}}$ & $22^{\mathrm{C}}$ & $24^{\mathrm{C}}$ & $10^{b}$ & $66^{\mathrm{e}}$ & $46^{d}$ & $64^{\mathrm{e}}$ \\
\hline Herbe fermentée & $0^{a}$ & $0^{a}$ & $0^{\mathrm{a}}$ & $0^{a}$ & $0^{a}$ & $0^{a}$ & $3^{a}$ & $12^{b}$ & $0^{a}$ \\
\hline Ensilage de maïs & $0^{a}$ & $0^{a}$ & $0^{a}$ & $1^{a}$ & $6^{a}$ & $3^{a}$ & $0^{a}$ & $11^{\mathrm{b}}$ & $1^{a}$ \\
\hline Complémentation en concentrés (\%) & $14^{a}$ & $14^{a}$ & $20^{b}$ & $25^{\mathrm{bc}}$ & $34^{\mathrm{d}}$ & $30^{\text {cd }}$ & $29^{\text {cd }}$ & $31^{\text {cd }}$ & $34^{d}$ \\
\hline \multicolumn{10}{|l|}{ Complémentation vitaminique } \\
\hline Vitamine A $\left(\times 10^{3}\right.$ Ul.vache $\left.{ }^{-1} .^{-1}\right)$ & $21^{a b}$ & $1,7^{\mathrm{a}}$ & $49^{b c}$ & $57^{\mathrm{bc}}$ & $60^{\mathrm{bc}}$ & $80^{\text {cd }}$ & $113^{d}$ & $87^{\mathrm{cd}}$ & $73^{\mathrm{cd}}$ \\
\hline Vitamine $E\left(U I . v^{2} e^{-1} . j^{-1}\right)$ & $35^{\mathrm{ab}}$ & $4^{a}$ & $80^{\mathrm{abc}}$ & $110^{\mathrm{bcd}}$ & $104^{\text {bcd }}$ & $166^{d}$ & $71^{\mathrm{abc}}$ & $138^{\mathrm{cd}}$ & $122^{\mathrm{cd}}$ \\
\hline \multicolumn{10}{|l|}{ Race $(\%)^{2}$} \\
\hline Abondance & $81^{d}$ & $7^{\mathrm{a}}$ & $44^{\mathrm{bc}}$ & $64^{\text {cd }}$ & $27^{\mathrm{ab}}$ & $29^{\mathrm{ab}}$ & $67^{\text {cd }}$ & $23^{\mathrm{ab}}$ & $69^{\text {cd }}$ \\
\hline Holstein & $0^{\mathrm{a}}$ & $3^{a}$ & $10^{\mathrm{a}}$ & $8^{a}$ & $34^{\mathrm{c}}$ & $28^{\mathrm{bc}}$ & $15^{\mathrm{ab}}$ & $14^{\mathrm{ab}}$ & $10^{\mathrm{a}}$ \\
\hline Montbéliarde & $19^{\mathrm{ab}}$ & $56^{d}$ & $42^{\mathrm{bcd}}$ & $27^{a b}$ & $30^{\mathrm{abc}}$ & $20^{\mathrm{ab}}$ & $16^{a}$ & $52^{\text {cd }}$ & $19^{a b}$ \\
\hline Tarentaise & $0^{a}$ & $34^{\mathrm{b}}$ & $2^{a}$ & $2^{a}$ & $2^{a}$ & $6^{a}$ & $2^{a}$ & $7^{\mathrm{a}}$ & $0^{\mathrm{a}}$ \\
\hline \multicolumn{10}{|l|}{ Stade de lactation } \\
\hline Stade moyen du troupeau (mois) & $7,5^{f}$ & $6,5^{\mathrm{e}}$ & $5,6^{\text {cd }}$ & $6,1^{\text {de }}$ & $5,4^{b c}$ & $5,4^{b c}$ & $4,1^{a}$ & $5,0^{b}$ & $5,0^{b}$ \\
\hline Vaches en début de lactation $(\%)^{2,3}$ & $6^{\mathrm{a}}$ & $13^{\mathrm{ab}}$ & $18^{\mathrm{bcd}}$ & $15^{\mathrm{abc}}$ & $24^{\text {cd }}$ & $14^{\mathrm{abc}}$ & $52^{\mathrm{e}}$ & $28^{d}$ & $25^{\mathrm{d}}$ \\
\hline Vaches en fin de lactation $(\%)^{2,4}$ & $66^{\mathrm{a}}$ & $44^{d}$ & $31^{\mathrm{bc}}$ & $37^{\mathrm{cd}}$ & $30^{\mathrm{abc}}$ & $25^{\mathrm{ab}}$ & $23^{a b}$ & $25^{\mathrm{ab}}$ & $20^{\mathrm{a}}$ \\
\hline Proportion de primipares (\%) ${ }^{2}$ & $16^{a}$ & $17^{a}$ & $18^{\mathrm{ab}}$ & $21^{\mathrm{abc}}$ & $24^{\mathrm{bc}}$ & $23^{\mathrm{abc}}$ & $20^{\mathrm{abc}}$ & $26^{\mathrm{c}}$ & $21^{\mathrm{abc}}$ \\
\hline Niveau de production (L.vache ${ }^{-1} \cdot \mathrm{j}^{-1}$ ) & $14^{a}$ & $15^{\mathrm{a}}$ & $18^{\mathrm{bc}}$ & $18^{\mathrm{bc}}$ & $19^{c}$ & $19^{c}$ & $16^{\mathrm{ab}}$ & $19^{c}$ & $19^{c}$ \\
\hline \multicolumn{10}{|l|}{ Acides gras ( $\%$ des AG totaux $)^{5}$} \\
\hline Saturés & $63^{a}$ & $66^{\mathrm{b}}$ & $67^{b}$ & $68^{\mathrm{b}}$ & $69^{c}$ & $70^{c}$ & $69^{c}$ & $73^{d}$ & $74^{d}$ \\
\hline $\mathrm{C} 4: 0$ & $4,0^{\mathrm{a}}$ & $4,1^{\mathrm{a}}$ & $4,2^{a}$ & $4,2^{\mathrm{a}}$ & $4,2^{\mathrm{a}}$ & $4,6^{\mathrm{d}}$ & $4,1^{a}$ & $4,0^{a}$ & $4,1^{\mathrm{a}}$ \\
\hline $\mathrm{C} 6: 0-\mathrm{C} 10: 0$ & $5,8^{a}$ & $7,4^{b}$ & $7,8^{b}$ & $7,5^{\mathrm{b}}$ & $7,8^{\mathrm{b}}$ & $8,9^{\mathrm{d}}$ & $7,3^{b}$ & $8,3^{c}$ & $8,5^{\mathrm{cd}}$ \\
\hline C12:0-C16:0 & $39^{a}$ & $42^{b}$ & $42^{b}$ & $43^{b c}$ & $44^{\text {cd }}$ & $45^{\mathrm{d}}$ & $44^{\mathrm{cd}}$ & $48^{e}$ & $49^{e}$ \\
\hline C18:0 & $9,1^{\mathrm{c}}$ & $8,6^{\mathrm{bc}}$ & $8,9^{\mathrm{c}}$ & $8,6^{\mathrm{bc}}$ & $8,6^{\mathrm{bc}}$ & $8,0^{b}$ & $9,0^{\mathrm{C}}$ & $8,0^{b}$ & $7,2^{\mathrm{a}}$ \\
\hline Monoinsaturés & $30^{e}$ & $27^{d}$ & $27^{d}$ & $27^{c d}$ & $25^{\mathrm{bc}}$ & $24^{b}$ & $26^{\text {cd }}$ & $23^{a}$ & $22^{a}$ \\
\hline $\mathrm{C} 18: 1 c 9(\mathrm{n}-9)$ & $20^{e}$ & $18^{\text {cd }}$ & $18^{\text {cd }}$ & $18^{\text {cd }}$ & $17^{\mathrm{bc}}$ & $16^{b}$ & $19^{d}$ & $16^{\mathrm{b}}$ & $15^{\mathrm{a}}$ \\
\hline $\mathrm{C} 18: 1 t 11+t 10)$ & $3,5^{\mathrm{e}}$ & $3,3^{\text {de }}$ & $3,0^{d}$ & $2,6^{\mathrm{c}}$ & $2,2^{b}$ & $2,1^{b}$ & $1,5^{\mathrm{a}}$ & $1,3^{\mathrm{a}}$ & $1,4^{\mathrm{a}}$ \\
\hline Polyinsaturés & $5,7^{d}$ & $5,3^{d}$ & $4,4^{\mathrm{C}}$ & $4,4^{c}$ & $3,9^{b}$ & $3,7^{a b}$ & $3,5^{\mathrm{ab}}$ & $3,3^{\mathrm{a}}$ & $3,5^{\mathrm{ab}}$ \\
\hline C18:2 c9c12 (n-6) & $1,7^{\mathrm{d}}$ & $1,6^{\text {ed }}$ & $1,3^{a b}$ & $1,5^{\mathrm{bc}}$ & $1,5^{\mathrm{c}}$ & $1,3^{\mathrm{a}}$ & $1,5^{\mathrm{c}}$ & $1,5^{\mathrm{bc}}$ & $1,6^{\mathrm{cd}}$ \\
\hline $\mathrm{C} 18: 2$ c9t11 (CLA) ${ }^{6}$ & $1,6^{\mathrm{d}}$ & $1,5^{\mathrm{d}}$ & $1,3^{\mathrm{c}}$ & $1,2^{\mathrm{c}}$ & $0,88^{b}$ & $0,93^{b}$ & $0,60^{\mathrm{a}}$ & $0,53^{a}$ & $0,58^{a}$ \\
\hline $\mathrm{C} 18: 3 c 9 c 12 c 15(\mathrm{n}-3)$ & $1,2^{f}$ & $1,0^{e}$ & $0,77^{\mathrm{bcd}}$ & $0,82^{\mathrm{d}}$ & $0,70^{\mathrm{abc}}$ & $0,67^{\mathrm{ab}}$ & $0,80^{\text {cd }}$ & $0,64^{a}$ & $0,75^{\mathrm{bcd}}$ \\
\hline Trans (CLA non inclus) & $5,9^{e}$ & $5,5^{\mathrm{de}}$ & $5,0^{\text {cd }}$ & $4,5^{\mathrm{c}}$ & $3,8^{b}$ & $3,8^{\mathrm{b}}$ & $2,8^{a}$ & $2,6^{\mathrm{a}}$ & $2,6^{\mathrm{a}}$ \\
\hline \multicolumn{10}{|l|}{ Vitamines et caroténoïdes ${ }^{7}$} \\
\hline$\beta$-Carotène (mg.kg $\left.{ }^{-1} \mathrm{MG}\right)$ & $3,5^{\mathrm{c}}$ & $5,8^{f}$ & $5,1^{\text {ef }}$ & $4,3^{d}$ & $4,6^{\mathrm{de}}$ & $5,5^{f}$ & $1,9^{\mathrm{ab}}$ & $2,3^{b}$ & $1,5^{\mathrm{a}}$ \\
\hline Xanthophylles (mg. $\left.\mathrm{kg}^{-1} \mathrm{MG}\right)$ & $0,31^{b}$ & $0,41^{\mathrm{c}}$ & $0,36^{\mathrm{bc}}$ & $0,25^{\mathrm{a}}$ & $0,24^{a}$ & $0,31^{b}$ & $0,21^{a}$ & $0,24^{a}$ & $0,20^{\mathrm{a}}$ \\
\hline Vitamine $\mathrm{A}$ (mg.kg $\left.{ }^{-1} \mathrm{MG}\right)$ & $5,0^{a b c}$ & $5,7^{\mathrm{c}}$ & $5,8^{\mathrm{C}}$ & $5,3^{b c}$ & $5,7^{c}$ & $5,8^{\mathrm{c}}$ & $4,5^{\mathrm{ab}}$ & $4,4^{\mathrm{a}}$ & $5,0^{\mathrm{abc}}$ \\
\hline Vitamine $\mathrm{E}\left(\mathrm{mg} \cdot \mathrm{kg}^{-1} \mathrm{MG}\right)$ & $8,0^{\text {cd }}$ & $9,3^{d}$ & $8,0^{\text {cd }}$ & $7,1^{\mathrm{bc}}$ & $6,3^{\mathrm{b}}$ & $8,1^{\text {cd }}$ & $3,9^{\mathrm{a}}$ & $3,9^{\mathrm{a}}$ & $3,2^{\mathrm{a}}$ \\
\hline Pot. antiox. $\left(\mathrm{mol} \mathrm{Fe}{ }^{2+} \cdot \mathrm{kg}^{-1} \mathrm{MS}\right)^{8}$ & $14^{\mathrm{bcd}}$ & $15^{\mathrm{de}}$ & $15^{\mathrm{de}}$ & $14^{\text {cde }}$ & $14^{\mathrm{bcd}}$ & $16^{\mathrm{e}}$ & $13^{\mathrm{bc}}$ & $12^{\mathrm{ab}}$ & $10^{\mathrm{a}}$ \\
\hline
\end{tabular}

$1 \%$ des matières sèches totales ingérées. $2 \%$ du troupeau. ${ }^{3}$ En lactation depuis moins de 2 mois. ${ }^{4}$ En lactation depuis plus de 7 mois. ${ }^{5}$ AG : acides gras. ${ }^{6}$ CLA : acide linoléique conjugué. ${ }^{7} \mathrm{MG}$ : matières grasses. ${ }^{8}$ Pot. antiox. : potentiel antioxydant. MS : matières sèches. ${ }^{a, b, c, d, e, f}$ Les valeurs ne présentant aucune lettre en commun diffèrent significativement $(P<0,05)$. 
Tableau 7. Typologie des fromages au lait de chèvre : caractéristiques nutritionnelles moyennes des classes de fromages présentées dans la figure 4 et conditions de production du lait associées (Lucas 2005).

\begin{tabular}{|c|c|c|c|c|c|c|c|c|}
\hline Classes de fromages & $\mathbf{I}$ & II & III & IV & $\mathbf{V}$ & VI & VII & VIII \\
\hline Nombre de fromages & 17 & 11 & 10 & 13 & 18 & 25 & 10 & 22 \\
\hline \multicolumn{9}{|l|}{ Ration fourragère (\%) ${ }^{1}$} \\
\hline Pâturage & $18^{\mathrm{b}}$ & $41^{\mathrm{c}}$ & $59^{d}$ & $33^{c}$ & $1^{\mathrm{a}}$ & $4^{\mathrm{a}}$ & $0^{\mathrm{a}}$ & $1^{\mathrm{a}}$ \\
\hline Foin & $47^{\mathrm{C}}$ & $30^{\mathrm{b}}$ & $14^{\mathrm{a}}$ & $35^{\mathrm{b}}$ & $60^{d}$ & $56^{c d}$ & $57^{\text {cd }}$ & $61^{\mathrm{d}}$ \\
\hline \multicolumn{9}{|l|}{ Concentrés } \\
\hline Niveau de complémentation $(\%)^{1}$ & $34^{\mathrm{bc}}$ & $29^{\mathrm{ab}}$ & $27^{\mathrm{a}}$ & $33^{\mathrm{abc}}$ & $39^{\text {cd }}$ & $40^{\text {cd }}$ & $43^{d}$ & $38^{\mathrm{cd}}$ \\
\hline Niveau de MG des concentrés $(\%)^{1}$ & $2,0^{d}$ & $1,1^{\mathrm{b}}$ & $0,7^{\mathrm{a}}$ & $1,1^{\mathrm{ab}}$ & $1,6^{\mathrm{c}}$ & $1,4^{\mathrm{bc}}$ & $1,4^{\mathrm{bc}}$ & $1,2^{\mathrm{bc}}$ \\
\hline Présence de lin (\% des fromages) & $100^{\mathrm{C}}$ & $55^{\mathrm{b}}$ & $10^{\mathrm{a}}$ & $23^{\mathrm{a}}$ & $44^{\mathrm{ab}}$ & $28^{\mathrm{ab}}$ & $10^{\mathrm{a}}$ & $23^{a}$ \\
\hline \multicolumn{9}{|l|}{ Complémentation vitaminique } \\
\hline Vitamine $A\left(\times 10^{3}\right.$ UI.chèvre $\left.{ }^{-1} \cdot j^{-1}\right)$ & 0 & 2,9 & 0,58 & 13 & 9,3 & 5,1 & 6,7 & 11 \\
\hline Vitamine E (Ul.chèvre $\left.{ }^{-1} \cdot j^{-1}\right)$ & 0 & 7 & 0 & 35 & 13 & 8 & 10 & 22 \\
\hline \multicolumn{9}{|l|}{ Race $(\%)^{2}$} \\
\hline Alpine & $46^{\mathrm{a}}$ & $72^{\mathrm{bc}}$ & $88^{\mathrm{c}}$ & $79^{\mathrm{bc}}$ & $83^{c}$ & $57^{\mathrm{ab}}$ & $92^{c}$ & $75^{\mathrm{bc}}$ \\
\hline Saanen & $54^{\mathrm{C}}$ & $28^{\mathrm{ab}}$ & $13^{a}$ & $21^{\mathrm{ab}}$ & $17^{\mathrm{a}}$ & $43^{\mathrm{bc}}$ & $8^{\mathrm{a}}$ & $25^{\mathrm{ab}}$ \\
\hline \multicolumn{9}{|l|}{ Stade de lactation } \\
\hline Stade moyen du troupeau (mois) & $5,1^{\mathrm{b}}$ & $6,1^{\mathrm{bc}}$ & $5,7^{\mathrm{bc}}$ & $7,6^{\mathrm{d}}$ & $3,3^{\mathrm{a}}$ & $4,9^{b}$ & $5,9^{\mathrm{bc}}$ & $6,9^{\mathrm{cd}}$ \\
\hline Chèvres en début de lactation $(\%)^{2,3}$ & $20^{\mathrm{ab}}$ & $9^{a b}$ & $5^{\mathrm{ab}}$ & $2^{\mathrm{a}}$ & $52^{c}$ & $25^{\mathrm{b}}$ & $19^{\mathrm{ab}}$ & $9^{\mathrm{ab}}$ \\
\hline Chèvres en fin de lactation $(\%)^{2,4}$ & $19^{\mathrm{ab}}$ & $33^{\mathrm{bcd}}$ & $26^{\mathrm{ab}}$ & $56^{\mathrm{d}}$ & $5^{\mathrm{a}}$ & $16^{\mathrm{ab}}$ & $30^{\mathrm{abc}}$ & $52^{\mathrm{cd}}$ \\
\hline Proportion de primipares $(\%)^{2}$ & $26^{\mathrm{b}}$ & $22^{\mathrm{ab}}$ & $23^{\mathrm{ab}}$ & $23^{\mathrm{ab}}$ & $24^{\mathrm{ab}}$ & $24^{\mathrm{ab}}$ & $15^{\mathrm{a}}$ & $24^{\mathrm{ab}}$ \\
\hline Niveau de production $\left(\right.$ L.chèvre $^{-1} \cdot \mathrm{j}^{-1}$ ) & $2,5^{\mathrm{d}}$ & $2,4^{\mathrm{cd}}$ & $2,0^{\mathrm{bc}}$ & $1,8^{\mathrm{ab}}$ & $2,6^{\text {cd }}$ & $2,3^{\text {cd }}$ & $1,6^{\mathrm{a}}$ & $1,7^{\mathrm{ab}}$ \\
\hline \multicolumn{9}{|l|}{ Acides gras (\% des AG totaux) } \\
\hline Saturés & $69^{\mathrm{a}}$ & $74^{\text {cd }}$ & $76^{\mathrm{de}}$ & $77^{\mathrm{e}}$ & $71^{\mathrm{b}}$ & $72^{\mathrm{bc}}$ & $73^{\mathrm{bc}}$ & $74^{\mathrm{cd}}$ \\
\hline $\mathrm{C} 4: 0$ & $2,8^{a}$ & $2,8^{\mathrm{a}}$ & $3,1^{\mathrm{b}}$ & $2,6^{\mathrm{a}}$ & $2,7^{\mathrm{a}}$ & $2,7^{\mathrm{a}}$ & $2,7^{\mathrm{a}}$ & $2.7^{\mathrm{a}}$ \\
\hline C6:0-C10:0 & $15^{\mathrm{a}}$ & $18^{\mathrm{cd}}$ & $20^{\mathrm{e}}$ & $18^{\mathrm{d}}$ & $16^{\mathrm{ab}}$ & $16^{\mathrm{ab}}$ & $16^{\mathrm{bc}}$ & $17^{\mathrm{cd}}$ \\
\hline C12:0-C16:0 & $34^{a}$ & $39^{\mathrm{bc}}$ & $39^{\mathrm{bc}}$ & $44^{\mathrm{d}}$ & $37^{\mathrm{b}}$ & $39^{\mathrm{bc}}$ & $40^{\mathrm{bc}}$ & $41^{c}$ \\
\hline C18:0 & $13^{\mathrm{e}}$ & $10^{\text {cd }}$ & $10^{\mathrm{bcd}}$ & $8,2^{\mathrm{a}}$ & $11^{\mathrm{d}}$ & $10^{\text {cd }}$ & $9,7^{b c}$ & $8.7^{\mathrm{ab}}$ \\
\hline Monoinsaturés & $25^{f}$ & $20^{\mathrm{bc}}$ & $19^{\mathrm{ab}}$ & $18^{a}$ & $23^{\text {ef }}$ & $23^{\text {de }}$ & $22^{\text {cde }}$ & $21^{\mathrm{cd}}$ \\
\hline C18:1 c9 (n-9) & $20^{\mathrm{e}}$ & $16^{\mathrm{ab}}$ & $15^{\mathrm{a}}$ & $14^{\mathrm{a}}$ & $18^{\mathrm{de}}$ & $18^{\text {de }}$ & $18^{\mathrm{cd}}$ & $17^{\mathrm{bc}}$ \\
\hline $\mathrm{C} 18: 1 t 11(+t 10)$ & $1,6^{\mathrm{c}}$ & $1,2^{\mathrm{b}}$ & $1,2^{b}$ & $0,87^{\mathrm{a}}$ & $1,0^{\mathrm{a}}$ & $0,95^{\mathrm{a}}$ & $0,95^{\mathrm{a}}$ & $0.91^{a}$ \\
\hline Polyinsaturés & $5,1^{d}$ & $4,4^{c}$ & $3,6^{\mathrm{a}}$ & $3,5^{\mathrm{a}}$ & $4,3^{c}$ & $4,2^{\mathrm{bc}}$ & $4,0^{\mathrm{abc}}$ & $3.8^{\mathrm{ab}}$ \\
\hline $\mathrm{C} 18: 2 \mathrm{c} 9 \mathrm{c} 12(\mathrm{n}-6)$ & $2,6^{\mathrm{e}}$ & $2,1^{\mathrm{bc}}$ & $1,8^{\mathrm{a}}$ & $1,9^{\mathrm{ab}}$ & $2,4^{\mathrm{de}}$ & $2,4^{\mathrm{de}}$ & $2,3^{d}$ & $2.2^{\mathrm{cd}}$ \\
\hline C18:2 c9t11 (CLA) & $0,56^{\mathrm{b}}$ & $0,44^{\mathrm{a}}$ & $0,42^{a}$ & $0,35^{\mathrm{a}}$ & $0,35^{\mathrm{a}}$ & $0,37^{\mathrm{a}}$ & $0,38^{\mathrm{a}}$ & $0.40^{a}$ \\
\hline $\mathrm{C} 18: 3 c 9 c 12 c 15(\mathrm{n}-3)$ & $1,0^{\mathrm{c}}$ & $0,81^{b}$ & $0,69^{\mathrm{ab}}$ & $0,57^{\mathrm{a}}$ & $0,82^{\mathrm{b}}$ & $0,73^{\mathrm{ab}}$ & $0,60^{\mathrm{a}}$ & $0.60^{a}$ \\
\hline Trans (CLA non inclus) & $4,4^{d}$ & $3,6^{c}$ & $3,2^{b c}$ & $2,6^{\mathrm{a}}$ & $3,2^{\mathrm{bc}}$ & $2,9^{\mathrm{ab}}$ & $2,9^{\mathrm{ab}}$ & $2.7^{\mathrm{ab}}$ \\
\hline \multicolumn{9}{|l|}{ Vitamines et caroténoödes ${ }^{7}$} \\
\hline Xanthophylles (mg.kg-1 MG) & $0,18^{\mathrm{a}}$ & $0,17^{\mathrm{a}}$ & $0,26^{\mathrm{b}}$ & $0,14^{\mathrm{a}}$ & $0,13^{\mathrm{a}}$ & $0,13^{\mathrm{a}}$ & $0,19^{\mathrm{a}}$ & $0.13^{a}$ \\
\hline Vitamine $\mathrm{A}\left(\mathrm{mg} \cdot \mathrm{kg}^{-1} \mathrm{MG}\right)$ & $6,8^{\mathrm{b}}$ & $7,0^{\mathrm{b}}$ & $9,3^{\mathrm{c}}$ & $6,8^{\mathrm{b}}$ & $4,9^{\mathrm{a}}$ & $6,9^{\mathrm{b}}$ & $4,8^{\mathrm{a}}$ & $6.0^{\mathrm{ab}}$ \\
\hline Vitamine $\mathrm{E}$ (mg.kg $\left.{ }^{-1} \mathrm{MG}\right)$ & $7,6^{b}$ & $7,9^{b}$ & $11^{\mathrm{c}}$ & $7,1^{\mathrm{b}}$ & $4,9^{\mathrm{a}}$ & $5,2^{\mathrm{a}}$ & $4,4^{\mathrm{a}}$ & $4.6^{\mathrm{a}}$ \\
\hline Pot. antiox. $\left(\mathrm{mol} \mathrm{Fe}^{2+} \cdot \mathrm{kg}^{-1} \mathrm{MS}\right)^{8}$ & $10^{\mathrm{ab}}$ & $16^{c}$ & $16^{\mathrm{c}}$ & $13^{\mathrm{bc}}$ & $9,4^{\mathrm{a}}$ & $9,8^{\mathrm{a}}$ & $11^{\mathrm{ab}}$ & $11^{\mathrm{ab}}$ \\
\hline
\end{tabular}

$1 \%$ des matières sèches totales ingérées. $2 \%$ du troupeau. 3 En lactation depuis moins de 2 mois. 4 En lactation depuis plus de 7 mois. 5 AG : acides gras. ${ }^{6}$ CLA : acide linoléique conjugué. ${ }^{7}$ MG : matières grasses. ${ }^{8}$ Pot. antiox. : potentiel antioxydant. MS : matières sèches. ${ }^{a, b, c, d, e, f}$ Les valeurs ne présentant aucune lettre en commun diffèrent significativement $(P<0,05)$.

nature de la rations de base. Il existe ainsi une forte opposition entre les fromages associés aux rations à base de pâturage (groupes I à VI) et ceux associés aux rations à base de fourrages conservés (groupes VII à IX). Les fromages associés au pâturage sont les plus riches en $\mathrm{AG}$ à longue chaîne, caroténoïdes, vitamine A et vitamine E, les plus pauvres en $A G$ saturés à moyenne chaîne et ceux qui ont le potentiel antioxydant le plus important. $\mathrm{Au}$ sein des groupes associés aux rations à base de pâturage, les variations de la composition du fromage sont liées essentiellement à des variations de la nature des prairies pâturées (alpage $v s$ prairies permanente ou temporaire), du niveau de complémentation en concentrés, de la race des animaux et de leur niveau de production. Par exemple, les fromages du groupe I, plus riches en $\mathrm{AG}$ à longue chaîne et moins riches en micronutriments liposolubles que les autres groupes de fromages associés au pâturage, sont associés à un pâturage en alpage, un faible niveau de complémentation en concentrés et des animaux majoritairement de race Abondance, caractérisés par un faible niveau de production. De même, les fromages du groupe $\mathrm{V}$, relativement riches en AG saturés à courte et moyen- 
ne chaîne et pauvres en $A G$ à longue chaîne et en micronutriments liposolubles au regard des autres groupes de fromages associés au pâturage, sont associés à un faible niveau de pâturage, un fort niveau de concentrés, des proportions significatives de prairies temporaires pâturées et d'animaux de race Holstein, une proportion de primipares dans le troupeau plus importante, indiquant un fort taux de renouvellement, et un niveau de production plus élevé. Le niveau d'intensification du système de production semble donc avoir un réel impact sur les caractéristiques nutritionnelles du fromage. Au sein des groupes de fromages associés aux rations à base de fourrages conservés, les variations de la composition du fromage semblent dépendre essentiellement du stade de lactation des animaux. Les fromages du groupe VII, particulièrement riches en $\mathrm{AG}$ à longue chaîne et pauvres en $\mathrm{AG}$ saturés à courte et moyenne chaîne, sont associés à une forte proportion d'animaux en début de lactation. Des mises bas regroupées en période hivernale semblent donc atténuer les variations de la composition du fromage liées à la nature de l'alimentation des animaux.

Sur le Rocamadour, 8 groupes de fromages ont été constitués. Ces groupes se distinguent autant par le profil en AG que par les teneurs en micronutriments liposolubles $\mathrm{du}$ fromage (figure 4). Cependant, contrairement aux fromages au lait de vache, il existe très peu d'associations entre ces deux familles de nutriments, ce qui suggère que, dans la gamme de conditions de production du lait étudiée, ces composés dépendent de facteurs de production différents. Les variations des teneurs en micronutriments du Rocamadour sont liées à des variations du mode de conservation des fourrages (pâturage $v s$ foin) et dans une moindre mesure du niveau de complémentation en concentrés (tableau 7). Ainsi, les fromages des groupes I à IV, les plus riches en ces micronutriments liposolubles, sont associés à des proportions de pâturage plus importantes et à des niveaux de complémentation en concentrés plus faibles. En revanche, le profil en AG $\mathrm{du}$ Rocamadour varie essentiellement avec la proportion de MG apportées par les concentrés dans la ration, la présence ou non de lin dans les concentrés et le stade de lactation des animaux. Par exemple, les fromages des groupes I et $\mathrm{V}$, particulièrement riches en $\mathrm{AG}$ à longue chaîne, sont associés à une forte proportion de MG apportées par les concentrés dans la ration, à une fréquence élevée de distribution de lin, ainsi qu'à une proportion de chèvres en début de lactation particulièrement importante. A l'inverse, les fromages des groupes IV et VIII, relativement pauvres en $\mathrm{AG}$ à longue chaîne, sont associés à une faible proportion de $\mathrm{MG}$ apportées par les concentrés dans la ration, à une faible fréquence de distribution de lin et à une faible proportion de chèvres en début de lactation.

\section{Conclusions et perspectives}

D'une manière générale, cette étude a contribué à enrichir les connaissances sur les caractéristiques nutritionnelles du fromage. Des données représentatives et détaillées sur sa composition en acides gras, vitamines, caroténoïdes et minéraux ont été collectées. Ce travail a aussi mis en évidence l'existence d'une forte variabilité de composition dans les fromages fermiers. D'autre part, les principaux facteurs de production responsables de cette variabilité de composition ont été identifiés et hiérarchisés. Ce travail a ainsi permis de montrer que la composition du fromage en $A G$ et en micronutriments liposolubles dépend de façon importante des conditions de production du lait, ce qui n'est pas le cas pour les minéraux et la vitamine B9 dont les teneurs dans le fromage sont fortement influencées par la technologie utilisée lors de la transformation fromagère. Les principaux facteurs de production du lait qui déterminent le profil en AG et les teneurs en micronutriments liposolubles du fromage ont été identifiés et les effets respectifs de ces facteurs de production sur la composition du fromage en ces composés ont été quantifiés. Chez la vache comme chez la chèvre, le rôle majeur de l'alimentation à base de fourrages pâturés sur les teneurs en micronutriments liposolubles des fromages à été mise en évidence. Chez la vache, le profil en AG des fromages a d'abord été lié à la nature de la ration de base, alors que chez la chèvre il a principalement dépendu de la nature des concentrés et du stade de lactation des animaux. Cette différence de résultats entre espèces est davantage due à des différences de pratiques d'élevage entre les vaches et les chèvres, concernant notamment la répartition des mises bas et la nature des concentrés, qu'à des effets différents des facteurs de production selon l'espèce animale. Ce travail a également montré, à travers la réalisation de typologies de fromages, que selon les conditions de production du lait, le fromage présente différents profils nutritionnels dont certains semblent être plus favorables pour la santé que d'autres.

L'approche corrélationnelle adoptée au cours de ce travail ne permet cependant pas de conclure formellement à des relations de causalité entre les conditions de production du lait et les teneurs en composés d'intérêt nutritionnel dans le fromage. En effet, il ne peut pas être exclu que certains résultats observés soient la conséquence d'effets de confusion liés à l'existence d'associations plus ou moins fortes entre pratiques d'élevage. Néanmoins, nous nous sommes en partie affranchis de cette limite en analysant un grand nombre d'échantillons. Les effets majeurs observés et en accord avec un nombre conséquent de résultats expérimentaux peuvent donc être considérés comme valides. C'est le cas par exemple des effets du niveau de pâturage, de la supplémentation lipidique et du stade de lactation. En revanche, certains effets mineurs demandent à être vérifiés en conditions expérimentales tels que ceux du stade de maturité et de la composition botanique de l'herbe ou de la race des animaux. Pour mieux comprendre les variations de la composition du fromage, certains aspects nécessitent également d'être approfondis, tels que les facteurs de variation du niveau de synthèse microbienne en vitamine B9 au cours de la fabrication fromagère ou la composition des fourrages en $\mathrm{AG}$, vitamines et caroténoïdes selon leur mode de conservation, leur composition botanique ou leur stade de maturité. Les résultats de ce travail devront d'autre part être élargis, par des dispositifs allégés, à d'autres variétés fromagères (en particulier les fromages persillés), d'autres espèces animales (brebis) et d'autres systèmes de production. Il apparaît également nécessaire de déterminer à présent, par des études d'intervention nutritionnelle sur modèle animal voire chez l'homme, dans quelle mesure les variations des caractéristiques nutritionnelles du fromage peuvent avoir une influence sur la santé humaine.

S'agissant des retombées pour les partenaires professionnels, les données collectées au cours de ce travail permettront tout d'abord de compléter les tables de composition des aliments. Cette étude pourra également servir dans l'avenir d'outil de réflexion sur 
l'évolution du cahier des charges des fromages sous signe de qualité. Enfin, si les résultats de ce travail ne peuvent être formellement généralisés à l'ensemble des fromages existants, ils permettront de développer une méthodologie pour caractériser d'autres variétés fromagères à travers un dispositif allégé.

\section{Remerciements}

Ce travail a été réalisé dans le cadre des programmes de recherche et développement du GIS Alpes du Nord et du Pôle Fromager AOC Massif Central. Nous tenons à remercier le Syndicat Interprofessionnel du Fromage d'Abondance, le Syndicat Interprofessionnel de la Tomme de Savoie, le Comité Interprofessionnel des Fromages du Cantal et le Syndicat des Producteurs du Fromage Rocamadour, ainsi que les 74 producteurs fermiers qui ont permis sa réalisation. Ce travail a par ailleurs bénéficié du soutien financier du FNADT, dans le cadre de la convention interrégionale de Massif : MassifCentral.

\section{Références}

AFSSA, 2002. Rapport du groupe de travail sur le sel. Agence Française de Sécurité Sanitaire des Aliments, Maisons-Alfort, France, 86p.

AFSSA, 2005. Risque et bénéfices pour la santé des acides gras trans apportés par les aliments. Agence Française de Sécurité Sanitaire des Aliments, Maisons-Alfort, France, 43p.

Agabriel C., Ferlay A., Journal C., Sibra C., Teissier D., Grolier, P., Bonnefoy J.C., Rock E., Chilliard Y., Martin B., 2004. Composés d'intérêt nutritionnel de laits de grand mélange : teneurs en acides gras et vitamines selon l'altitude et la saison. Renc. Rech. Rum., 11, 51-54.

Bossart M.A., Leuenberger H., Kuenzi N., Blum J.W., 1985. Levels of hormones and metabolites, insulin responses to glucose infusions, glucose tolerances and growth rates in different breeds of steers: studies during and after an alpine sojourn. Z. Tierzüchtg Züchtgsbiol., 102, 23-33.

Boufaïed H., Chouinard P.Y., Tremblay G.F., Petit H.V., Michaud R., Bélanger G., 2003. Fatty acids in forages. I. Factors affecting concentrations. Can. J. Anim. Sci., 63, 501-511.

Bugaud C., Bornard A., Hauwuy A., Martin B., Salmon J.C., Tessier L., Buchin S., 2000. Relation entre la composition botanique de végétations de montagne et leur composition en composés volatils. Fourrages, 162, 141-155.

Bugaud C., Buchin S., Coulon J.B., Hauwuy A., Dupont D., 2001. Influence of the nature of alpine pastures on plasmin activity, fatty acid and volatile compound composition of milk. Lait, 81, 401-414.

Chilliard Y., Ferlay A., Mansbridge R.M., Doreau M., 2000. Ruminant milk fat plasticity: nutritional control of saturated, polyunsaturated, trans and conjugated fatty acids. Ann. Zootech., $49,181-205$

Chilliard Y., Ferlay A., Rouel J., Lamberet G. 2003. A review of nutritional and physiological factors affecting goat milk lipid synthesis and lipolysis. J. Dairy Sci., 86, 1751-1770.

Chilliard Y., Lamberet G., 2001. Biochemical characteristics of goat milk lipids and lipolytic systems. A comparison with cow and human milk. Effects of lipid supplementation. In: Freund G (Eds), Recent advances on goat milk quality, raw material for cheesemaking, ITPLC Editions, Surgères, France, 71-114.

CNIEL, 2005. Centre National Inter-professionnel de l'Economie Laitière, Banque de données statistiques, http://www.cniel.com.

Collomb M., Bütikofer U., Sieber R., Bosset J.O., Jeangros B., 2001. Conjugated linoleic acid and trans fatty acid composition of cows' milk fat produced in lowlands and highlands. J. Dairy Res., 68, 519-523.
Darnton-Hill I., Nishida C., James W.P., 2004. A life course approach to diet, nutrition and the prevention of chronic diseases. Public Health Nutr., 7, 101-121.

ENITA, 2002. Analyse de la diversité des conditions de production au sein de 4 filières fromagères. Ecole Nationale d'Ingénieurs des Travaux Agricoles, Clermont-Ferrand, France, $1-36$.

Fedele V., Rubino R., Claps S., Manzi P., Marconi S., Pizzoferrato L., 2004. Seasonal variation in retinol concentration of goat milk associated with grazing compared to indoor feeding. South Afric. J. Anim. Sci., 34 (Suppl. 1) 148-150.

Guéguen L., 1971. La composition minérale du lait et son adaptation aux besoins minéraux du jeune. Ann. Nutr. Alim., 24, A335-A381.

Iwanska S., Pysera B., Strusinska D., 1997. Carotenoids content of green forages and preserved feeds. Acta Academia Agriculturae ac Technicae Olstenensis Zootechnica, 47, 117-128.

Jensen S.K., Johannsen A.K., Hermansen J.E., 1999. Quantitative secretion and maximal secretion capacity of retinol, beta-carotene and alphatocopherol into cows' milk. J. Dairy Res., 66, 511-522.

Krukovsky V.N., Whiting F., Loosli J.K., 1950. Tocopherol, carotenoid and vitamin A content of the milk fat and the resistance of milk to the development of oxidized flavors as influenced by breed and season. J. Dairy Sci., 33, 791-796.

Lawless F., Stanton C., L'Escop P., Devery R., Dillon P., Murphy J.J., 1999. Influence of breed on milk cis-9, trans-11-conjugated linoleic acid content. Livest. Prod. Sci., 62, 43-49.

Leiber F., Kreuzer M., Nigg D., Wettstein H.R., Schreeder M.R.L., 2005. A study on the causes for the elevated n-3 fatty acids in cows' milk of alpine origin. Lipids, 40, 191-202.

Lucas A., 2005. Relations entre les conditions de production du lait et les teneurs en composés d'intérêt nutritionnel dans le fromage. Thèse de doctorat, Université d'Auvergne, $181 \mathrm{p}$.

Lucas A., Agabriel C., Martin B., Ferlay A. Verdier-Metz I., Coulon J.B., Rock E., 2006a. Relationships between the conditions of cow's milk production and the contents of components of nutritional interest in raw milk farmhouse cheese. Lait, sous presse.

Lucas A., Rock E., Chamba J.F., Verdier-Metz I., Brachet P., Coulon J.B., 2006b. Respective effects of milk composition and the cheesemaking process on cheese compositional variability in components of nutritional interest. Lait, $86,1-21$.
Lucey J.A., Fox P.F., 1993. Importance of calcium and phosphate in cheese manufacture: a review. J. Dairy Sci., 76, 1714-1724.

Martin, A., 2001. Apports nutritionnels conseillés pour la population française, 3 ème $\mathrm{Ed}$. Lavoisier, Paris, France, 605 pp

Martin B., Ferlay A., Pradel P., Rock E., Grolier P., Dupont D., Gruffat D., Besle J.M., Ballot N., Chilliard Y., Coulon J.B., 2002. Variabilité de la teneur des laits en constituants d'intérêt nutritionnel selon la nature des fourrages consommés par les vaches laitières. Renc. Rech. Rum., 9, 347-350.

Mora O., Romano J.L., Gonzalez E., Ruiz F., Shimada A., 2000. Low cleavage activity of 15 , 15 ' dioxygénase to convert $b$ ? carotene to retinal in cattle compared with goats, is associated with the yellow pigmentation of adipose tissue. Int. J. Vitam. Nutr. Res., 70, 199-205.

Morand-Fehr P., Tran G., 2001. La fraction lipidique des aliments et les corps gras utilisés en alimentation animale. INRA Prod. Anim., 14 285-302.

Moreno-Rojas R., Amaro-Lopez M.A. Garcia-Gimeno R.H., Zurera-Cosano G., 1995. Effects of Manchego-type cheese-making process on contents of mineral elements. Food Chem., 53, 435-439.

Park Y.W., Anderson M.J., Walters J.L., Mahoney A.W., 1983. Effects of processing methods and agronomic variables on carotene contents in forages and predicting carotene in alfalfa hay with near-infrared-reflectance spectroscopy. J. Dairy Sci., 66, 235-245.

Robowsky K.D., Knabe O., 1972. Investigations on the alpha-tocopherol content of fodder grasses. 3. Influence of conservation and storage on the alpha-tocopherol content of fodder grasses. Archiv. fur Tierernahrung, 22, $125-$ 132

Shahani K.M., Hathaway I.L., Kelly P.L., 1962. B-complex vitamin content of cheese. II. Niacin, panthotenic acid, pyridoxine, biotin, and folic acid. J. Dairy Sci., 45, 833-841.

Sharma Y.K., Goyal K.C., Mistra U.K., 1983. Vitamin A and RBP contents of milk of goats given a single massive dose of vitamin A orally. Nutr. Rep. Int., 28, 593-596.

Shingfield K.J., Salo-Väänänen P., Pahkala E., Toivonen V, Jaakkola S, Piironen V, Huhtanen P., 2005. Effect of forage conservation method, concentrate level and propylene glycol on the fatty acid composition and vitamin content of cows' milk. J. Dairy Res., 72, 349-361.

Sollberger H., Schaeren W., Collomb M., Badertscher R., Bütikofer U., Sieber R., 2004 Beitrag zur kenntnis der zusammensetzung von 
ziegenmilch schweizerischer herkunft. ALP Sci., 473, 1-16.

Stockdale C.R., Walker G.P., Wales W.J., Dalley D.E., Birkett A., Shen Z., Doyle P.T., 2003. Influence of pasture and concentrates in the diet of grazing dairy cows on the fatty acid composition of milk. J. Dairy Res., 70, $267-$ 276.

Wong N.P., Lacroix D.E., Mattingly W.A., Vestal J.H., Alford J.A., 1976. The effect of manufacturing variables on the mineral content of Cottage cheese. J. Dairy Sci., 59, 41-44.
Yang A., Tume R.K., 1993. A comparison of $\beta$-carotene-splitting activity isolated from intestinal mucosa of pasture-grazed sheep, goats and cattle. Biochem. Mol. Biol. Int., 30, 209217.

\section{Résumé}

Les relations entre les conditions de production du lait et les teneurs en composés d'intérêt nutritionnel de fromages fermiers au lait de vache ou de chèvre ont été étudiées en conditions réelles de production au sein de 4 filières fromagères (Abondance, Tomme de Savoie, Salers/Cantal et Rocamadour). Au total, 432 fromages, dont 306 au lait de vache et 126 au lait de chèvre, produits tout au long de l'année par 74 producteurs fermiers ont été analysés. Dans un premier temps, les effets respectifs des conditions de production du lait et de la transformation fromagère sur la variabilité de composition du fromage ont été quantifiés. Dans un second temps, les relations entre la composition du fromage et les conditions de production du lait, préalablement identifiées au moyen d'enquêtes, ont été étudiées. Le fromage fermier se caractérise par une grande variabilité de composition qui dépend majoritairement de la transformation fromagère pour la vitamine $\mathbf{B} 9$ et les minéraux et des conditions de production du lait pour les acides gras (AG), les vitamines $\mathbf{A}$ et $\mathbf{E}$ et les caroténoïdes. L'espèce animale explique une grande partie de la variabilité en certains AG et en $\beta$-carotène dans le fromage. Les teneurs en micronutriments liposolubles et le potentiel antioxydant du fromage dépendent principalement de la nature de la ration fourragère (pâturage vs fourrages conservés) quelle que soit l'espèce animale. Les rations à base de pâturage sont associées à des teneurs en caroténoïdes et en vitamines $A$ et $\mathbf{E}$ et à un potentiel antioxydant dans le fromage plus importants. En revanche, les facteurs de variation du profil en AG du fromage ont différé dans cette étude entre les vaches et les chèvres. Pour les fromages au lait de vache, il dépend principalement de la nature de la ration fourragère (pâturage vs fourrages conservés). Les fromages associés au pâturage sont plus riches en AG saturés et insaturés à longue chaîne $(\geq \mathrm{C} 18)$ et plus pauvres en AG saturés à courte et moyenne chaîne (C6:0 à C16:0). Le profil en $A G$ du fromage au lait de chèvre dépend principalement de la proportion de matières grasses apportées par les concentrés dans la ration, du stade de lactation des animaux et dans une moindre mesure de la présence ou non de lin dans les concentrés. Ce fromage est plus riche en AG à longue chaîne et plus pauvre en AG saturés à courte et moyenne chaîne lorsque la proportion de matières grasses apportées par les concentrés est plus importante, les concentrés contiennent du lin et les animaux sont en début de lactation. En conclusion, ce travail a permis de collecter des données représentatives et détaillées sur les caractéristiques nutritionnelles de différentes variétés fromagères et de hiérarchiser les effets des principaux facteurs de production responsables de la variabilité de composition du fromage.

\section{Abstract}

\section{Relationships betwwen the conditions of milk production and the contents of nutritional constituents in cheese: study under real conditions of} production

The relationships between the conditions of milk production and the contents of components of nutritional interest in cow's or goat's milk farmhouse cheeses have been studied under real conditions of production in four cheese chains (Abondance, Tomme de Savoie, Salers/Cantal and Rocamadour). In total, 432 cheeses, including 306 cow's milk ones and 126 goat's milk ones, produced throughout the year by 74 farmhouse producers, were analysed. First, the respective effects of the conditions of milk production and the cheese-making process on the compositional variability in cheese were quantified. Subsequently, the relationships between the cheese composition and the conditions of milk production, beforehand identified by means of surveys, were studied. Farmhouse cheese was characterized by a great compositional variability which mainly depended on the cheese-making process for vitamin $B 9$ and minerals and on the conditions of milk production for fatty acids (FA), vitamins $A$ and $E$ and carotenoids. The animal species explained a great part of the variability in some FA and $\beta$-carotene contents in cheese. The contents of fat-soluble micronutrients and the antioxidant capacity in cheese mainly depended on the nature of the fodder ration (pasture $v s$. preserved forages) whatever the animal species. The pasture-based rations were associated with higher levels of carotenoids, vitamins $A$ and $E$ and antioxidant capacity in cheese. On the other hand, the influencing factors of the FA profile in cheese differed in this study between cows and goats. As regards cow's milk cheeses, it mainly depended on the nature of the fodder ration (pasture vs preserved forages). Cheeses associated with pasture were richer in long length saturated and unsaturated FA $(\geq \mathrm{C18})$ and poorer in short and medium length saturated FA (C6:0 à C16:0). The FA profile of goat's milk cheese mainly depended on the percentage of fat from the concentrates in the ration, the stage of lactation of animals and to a lesser extent the presence or not of linseed in the concentrates. This cheese was richer in long length FA and poorer in short and medium length saturated FA when the percentage of fat from the concentrates was higher, the concentrates contained linseed and the animals were in early lactation. In conclusion, this work allowed to collect representative and detailed data on the nutritional characteristics of various cheese varieties and to hierarchy the main production factors responsible for the compositional variability in cheese.

LUCAS A., HULIN S., MICHEL V., AGABRIEL C., CHAMBA J.-F., ROCK E., COULON J.-B., 2006. Relations entre les conditions de production du lait et les teneurs en composés d'intérêt nutritionnel dans le fromage : étude en conditions réelles de production. INRA Prod. Anim., 19, 15-28. 